\title{
Exploiting DLP Illumination Dithering for Reconstruction and Photography of High-speed Scenes
}

\author{
Sanjeev J. Koppal - Shuntaro Yamazaki - Srinivasa G. Narasimhan
}

Received: — / Accepted: -

\begin{abstract}
In this work, we recover fast moving scenes by exploiting the high-speed illumination "dithering" of cheap and easily available digital light processing (DLP) projectors. We first show how to reverse-engineer the temporal dithering for off-the-shelf projectors, using a high-speed camera. DLP dithering can produce temporal patterns commonly used in active vision techniques. Since the dithering occurs at a very high framerate, such illumination-based methods can be "sped up" for fast scenes. We demonstrate this with three applications, each of which only requires a single slide to be displayed by the DLP projector. The quality of the result is determined by the camera frame-rate available to the user. Pairing a high-speed camera and a DLP projector, we demonstrate structured light reconstruction at $100 \mathrm{~Hz}$. With the same camera and three or more DLP projectors, we show photometric stereo and demultiplexing applications at $300 \mathrm{~Hz}$. Finally, with a real-time $(60 \mathrm{~Hz})$ or still camera, we show that DLP illumination acts as a very fast flash, allowing strobe photography of high-speed scenes. We discuss, in depth, some characteristics of the temporal dithering with a case study of
\end{abstract}

This is an extension and consolidation of our previous work on the active vision systems using DLP projectors ([24],[16]).

Srinivasa G. Narasimhan and Sanjeev J. Koppal

The Robotics Institute, Carnegie Mellon University, USA

Tel.: +1-412-268-3818

Fax.: +1-412-268-6436

E-mail: srinivas@cs.cmu.edu,koppaldev@gmail.com

Shuntaro Yamazaki

National Institute of Advanced Industrial Science and Technology, Japan

Tel.: +81-3-3599-8358

Fax.: +81-3-5530-2066

E-mail: shun-yamazaki@aist.go.jp a particular projector. Finally, we describe limitations, trade-offs and other issues relating to this work.

Keywords Active Vision · DMD · DLP · High Speed Camera $\cdot$ Temporal Dithering

\section{Introduction}

Illumination changes create powerful visual cues. These are widely exploited in computer vision and graphics for scene analysis. Photometric lighting-based techniques first choose a reflectance model and then estimate its parameters from images taken under varying lighting. This is the basis for classical photometric stereo ([45]) for lambertian scenes, as well as several extensions for non-lambertian low parameter BRDFs. However, most of these methods rely on changes in light-source location or direction. Instead, there are other approaches that employ temporal or spatial modulation of the incident light-field. These techniques obtain correspondences between the light source and the camera to recover scene properties (such as scene depths [44]).

In this paper, we are interested in analyzing highspeed scenes, imaged at faster-than-real-time rates $(\geq$ $120 \mathrm{~Hz}$ ). Almost all of the correspondence-based lighting techniques described above avoid the difficult problem of tracking moving scene points. Instead, these require that the illumination modulation occurs at a much faster rate than scene motion. This means that during image capture, the scene must be essentially static. Most reasonably fast scenes quickly outpace physical methods of moving or modulating the light-source, such as handwaving or shifting attenuating masks.

This would suggest that high-speed scene analysis should be performed only with passive techniques, such as multi-view stereo, by using high-frame rate cameras. 
Although very expensive, today such cameras can capture video at speeds up to $10000 \mathrm{~Hz}$. However, two problems exist with passive methods. First, there are various types of scene information (such as high-resolution surface normals from photometric stereo, and directindirect components from fast separation [26]) which cannot be obtained purely by using cameras. Second, since camera motion would be much slower than scene motion, multiple viewpoints would be required. This would imply additional cameras, increasing the already prohibitive cost.

Therefore, in this work, we focus on speeding up active vision techniques for fast scenes. Our approach is to rely on electronic means of controlling illumination. Such computer controlled illumination would modulate the light much faster than the scene motion. One way to do this would be to use LCDs (liquid crystal displays) [49] which can modulate illumination at real time (60$120 \mathrm{~Hz}$ ) rates. However, for faster scenes, as those depicted in our work, most LCDs simply do not change quickly enough. In addition, at higher speeds, they exhibit SNR issues for image capture, since the incident light is attenuated.

Recently, a MEMS device called the Digital Micromirror Device (DMD) has been introduced to modulate illumination. Each mirror in the DMD is $14 \times 14$ microns and can switch between two orientations, $+12^{\circ}$ and $-12^{\circ}[8]$. In one orientation, incident light is reflected by the mirror toward the outside scene and in the other, light is reflected onto a black surface within the projector. These mirrors can switch between orientations in a few microseconds, enabling high precision control of illumination. As a result, the DMD device has found applications in areas ranging widely from microscopy to chemistry to holographic displays [8].

However, DMD kits that allow total control and programmability of the incident illumination are almost as expensive as high-speed cameras, making their use for high-speed scene analysis difficult. Our key insight was to note that an integral component of all Digital Light Processing (DLP) technology projectors (http://www.dlp.com) is also a DMD chip. Therefore DLP technology has enabled mass production of low cost DMD chips with one drawback: we cannot directly control the mirror states of the DMD chip. However, we can control the slides that the projector will emit. This paper investigates techniques to reverse-engineer and exploit cheap, off-the-shelf DLP projectors for fast active vision.

\subsection{Related work}

Projectors are commonly used as programmable light sources for a variety of active vision techniques including structured light reconstruction [44,48,7,6,47,37], photometry-based scene recovery $[52,11]$, relighting [43], light transport analysis $[26,39]$ and depth from defocus [50]. The intensity and color of the scene's illumination can be controlled over space and time depending on the task at hand. For instance, projecting a set of colored striped patterns onto a scene alleviates the problem of finding correspondences for 3D reconstruction [48].

The operating principle of the DMD device has also been exploited in computer vision and graphics. Nayar et al. [25] re-engineer a DLP projector into a DMDcamera and demonstrate the notion of programmable imaging for applications including adaptive dynamic range and optical filtering and matching. Based on the theory of compressive sampling, a single pixel camera has been implemented where the DMD device is used to compute optical projections of scene radiance [40]. Raskar et al [33] and Cotting et al [5] use cameraprojector synchronization to embed illumination patterns in the scene that cannot be observed with the naked eye. Jones et al. [14] modify a DLP projector using custom made FPGA-based circuitry to obtain 1-bit projection at $4800 \mathrm{~Hz}$. Using this, they generate high speed stereoscopic light field displays. McDowall and Bolas [20] use a specially re-programmed high speed projector based on Multiuse Light Engine (MULE) technology to achieve range finding at kilohertz rates.

Our work has the potential to extend any active vision technique to dynamic scenarios, and we illustrate this with a few popular applications, such as structured light reconstruction and photometric stereo. Since illumination dithering can be observed reliably even with camera frame rates as low as $300 \mathrm{fps}$, it enables applications with slower performance requirements. In addition, with still or $60 \mathrm{~Hz}$ cameras, we demonstrate highspeed photography applications inspired from computational photography techniques, such as shape time photography ([9]). Finally, unlike previous work, our techniques do not require any projector-camera synchronization, hardware modification/re-programming of the DMD device, or the knowledge of proprietary dithering coding schemes. Thus, we believe this work to be widely applicable. Better visualizations of all our results are available through our websites (http://www. cs.cmu.edu/ ILIM/projects/IL/dlp-dithering/, $[17],[18])$. 


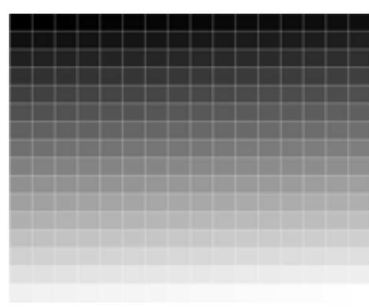

(b) Calibration image input to projector

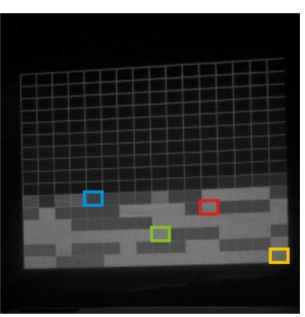

Frame 5

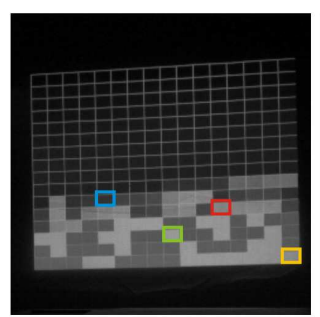

Frame 27

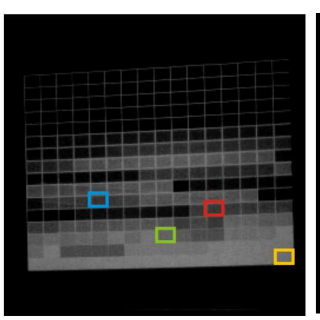

Frame 85

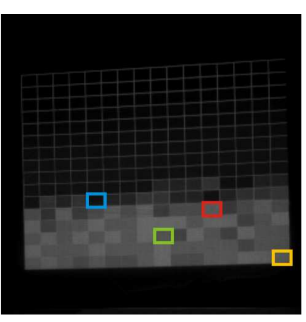

Frame 91

(c) Images measured by a high-speed camera

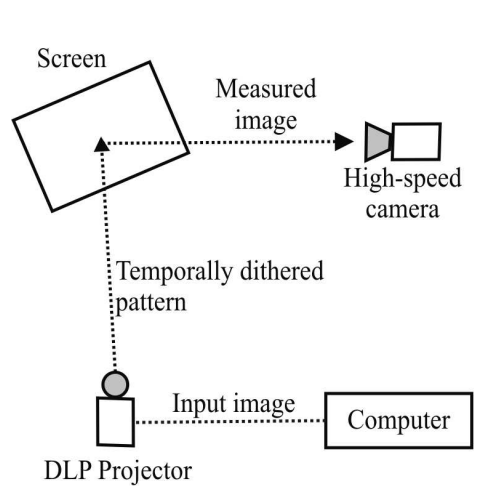

(a) Illustration

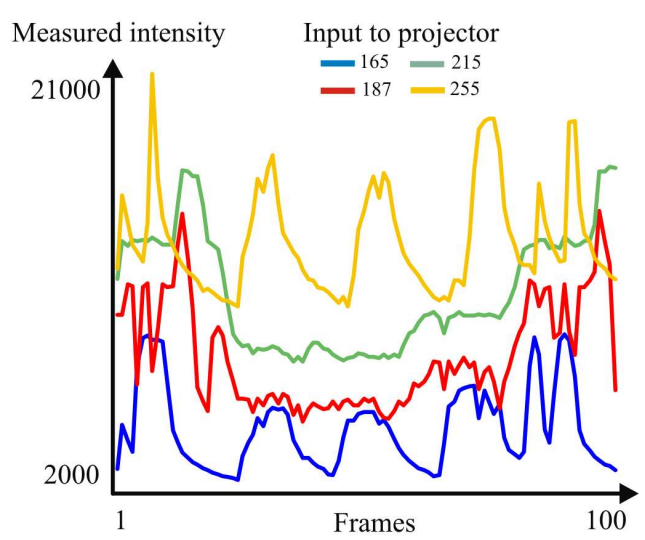

(d) Observed temporal dithering

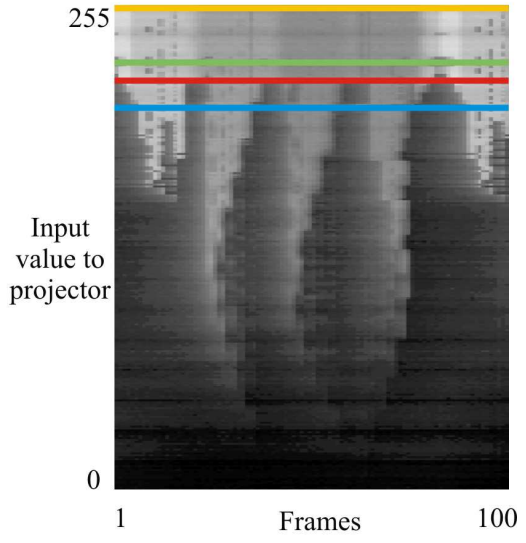

(e) Projector codes

Fig. 1 Reverse engineering a DLP Projector: (a) A DLP projector converts the input intensity received into a stream of light pulses that is then projected onto a screen. A high speed camera viewing the screen aggregates the brightness over the duration of its integration time. (b) An image composed of $5 \times 5$ pixel blocks each with a different intensity from 0 to 255 is input to the projector. (c) The camera records the projector output at $10 \mathrm{kHz}$. In (d) we show gray-valued intensities measured over time by the high speed camera for 4 example intensities input to the projector. Notice the significant variations in the plots. In (e), the temporal dithering for all 256 projector input intensities is collated into an image. This temporal dithering is repeatable and can be used to encode illumination in a novel way, enabling fast active vision.

\section{Temporal Dithering in a DLP projector}

In order to project a desired intensity value, the DLP projector emits a series of light pulses of different time intervals [8]. A sensor aggregates the pulses of light over the duration of its integration time (say, $1 / 30$ s in a video camera) to capture the final gray-valued brightness. This Pulse-Width modulation (PWM) by the projector is unique for every input intensity and can be termed as "temporal dithering" of the illumination. As we shall show, this dithering allows us to encode scene illumination in novel ways to achieve significant speedup in the performance of virtually any active vision technique.

But how do we capture this high speed dithering? The exposure time $(1 / 30$ s) of a video camera is too long to observe the temporal illumination dithering clearly. One possibility is to precisely synchronize the camera with a DLP projector and to expose the camera only for the duration of a single projected light pulse (for a few microseconds as in [5]). This would restrict us to applications with $30-60 \mathrm{~Hz}$ performance requirements.
In contrast, our work focuses on exploiting the temporal dithering for fast active vision. For this, we use a novel combination of a high speed camera and an offthe-shelf DLP projector. Figure 1 illustrates the dithering of an 8-bit InFocus IN38 DLP projector as observed by a Photron PCI-1024 high speed camera. An image composed of $5 \times 5$ pixel blocks each with a different intensity value from 0 to 255 is input to the projector. Each intensity at a pixel $C$ in this image is projected onto a flat screen using a unique temporal dithering $D_{C}(t)$, over discrete time frames $t$. The high speed camera observes the projected images at $10 \mathrm{kHz}$. Notice the significant variation in the images recorded. The plot in Figure 1(d) shows the temporal codes emitted by the projector for 4 input brightnesses (165, 187, 215, 255), as measured over 100 camera frames. The temporal ditherings corresponding to all the 256 input intensities are collated into a photograph for better visualization of this principle.

Temporal dithering occurring in a commercial projector is not the only effect measured by the profiles in Figure 1. The projector has a bulb that is never really 


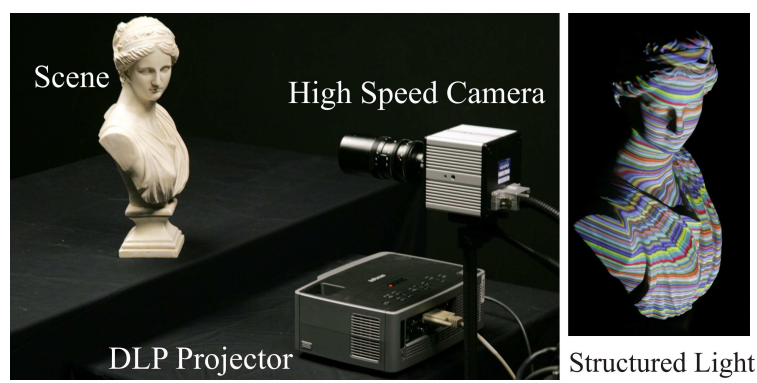

Fig. 2 Illumination and acquisition setup for structured light based 3D reconstruction: (a) The Photron high speed camera is placed vertically above the Infocus DLP projector in our acquisition setup. A vertical plane is placed at known distance behind the scene to enable finding correspondences. (b) The scene (statue) is illuminated using only a single pattern, as shown on the right. This pattern is static when viewed directly by unaided eyes. At high-frame rate, however, it is decomposed into a highfrequency patterns by the DLP projector.

"turned off" even when projecting black pixels. This is why all the projectors have a non-zero minima, and can be easily corrected by subtracting the ambient image due to a black slide. In addition, we use no synchronization, and therefore the different profiles are measured across different positions of the color-wheel (since measuring 100 frames at $10 \mathrm{kHz}$ requires a $\frac{1}{100}^{\text {th }}$ second, which is approximately a single color-wheel cycle). This explains the intensity shift in the profiles, which can be addressed by using a projector with the color-wheel removed (as in Section 6, [19], [4]). If those two calibration steps are taken, the profiles in the figure would closely resemble the binary "step function" codes we would expect ([8]). Any remaining differences are attributable to the spatial dithering imposed by the manufacturer (discussed in Section 7). These differences do not affect the use of structured light approaches, since the confusion matrices (depicting the closeness of the profiles to each other) are consistently diagonal, as shown in Fig. 3 and Fig. 17 and discussed in Section 7, where we also demonstrate the repeatability of these patterns.

The projected temporal dithering codes vary at about $10000 \mathrm{~Hz}$, which is faster than most dynamic scenes and, in our experience, is detectable by high-speed cameras with a frame-rate of 250fps or more. Consider a dynamic scene and a high-speed camera whose frame-rate is selected such that, over a short interval of measured frames, the scene appears essentially static. If the camera frame rate is above $250 \mathrm{fps}$, then the illumination in these frames will vary, even though the scene appears to have no motion.

This is exactly the assumption made by many active vision algorithms, and therefore, if we pick an appropriate pattern to project, we can extend these methods to dynamic scenes. In the next sections, we will exploit this aspect of DLP illumination to enable dynamic scene recovery and capture using well-known techniques such as structured light reconstruction, photometric stereo, illumination demultiplexing and strobe-light photography. In all our experiments (Sections 3 through 6), each projector emits a single slide and the selection of the slide pattern is done heuristically; for example, we select a random trio set of intensities for the three projectors in photometric stereo. Designing better patterns requires understanding different characteristics of the DLP illumination codes, and in Section 7 we do so for a particular projector/high-speed camera case. In that discussion, we also present a brute-force search method for selecting the pattern of the slide that the projector will display. We conclude with Section 8 that includes a description of the limitations, trade-offs and other issues relating to this work.

\section{3D Shape Acquisition}

Structured light-based triangulation has commonly been used for 3D reconstruction [44,31,1]. A known set of patterns that change their intensity spatially and/or temporally is projected onto a scene and the reflected images are used to find the corresponding pixels between the projector and the camera [35]. The corresponding pixels are then triangulated spatially to obtain 3D structure. It is assumed that the scene motion is negligible or sufficiently small [42] while the patterns are projected. Since projectors have been operated at $30-60 \mathrm{~Hz}$, most implementations achieve slower than real-time performances. Using these same algorithms with the fast illumination dithering in a DLP projector should enable high speed reconstruction.

Our goal is to obtain correspondences from camera pixels to projector pixels at high speeds. For convenience, the camera and the projector are placed in a fronto-parallel configuration with a vertical baseline (see Figure 2). The high speed camera is operated at $3 \mathrm{kHz}$ and the projector is reverse engineered at this speed as described in Section 2. We assume that the camera and the projector are geometrically calibrated in advance. In our experiments, the intrinsic parameters of a camera are estimated by using a planar calibration pattern [51], and the other parameters are determined using the homographies between the image planes of camera and projector [34].

\subsection{Illumination Pattern}

The differences the among existing techniques of structured light-based triangulation lie in the illumination 


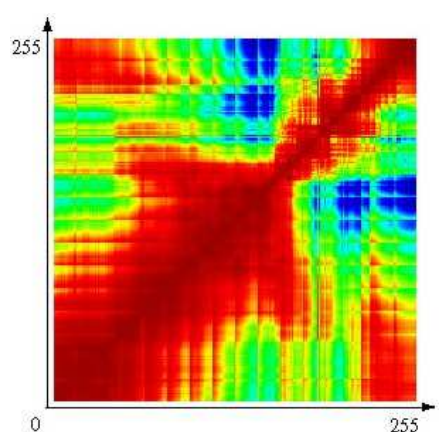

(a) dot-product of intensity

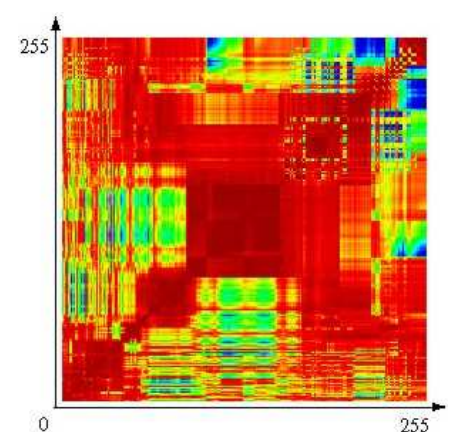

(b) worst match of gradient

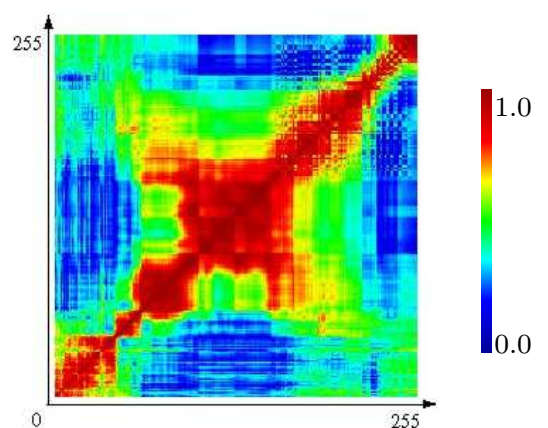

(c) dot-product of gradient

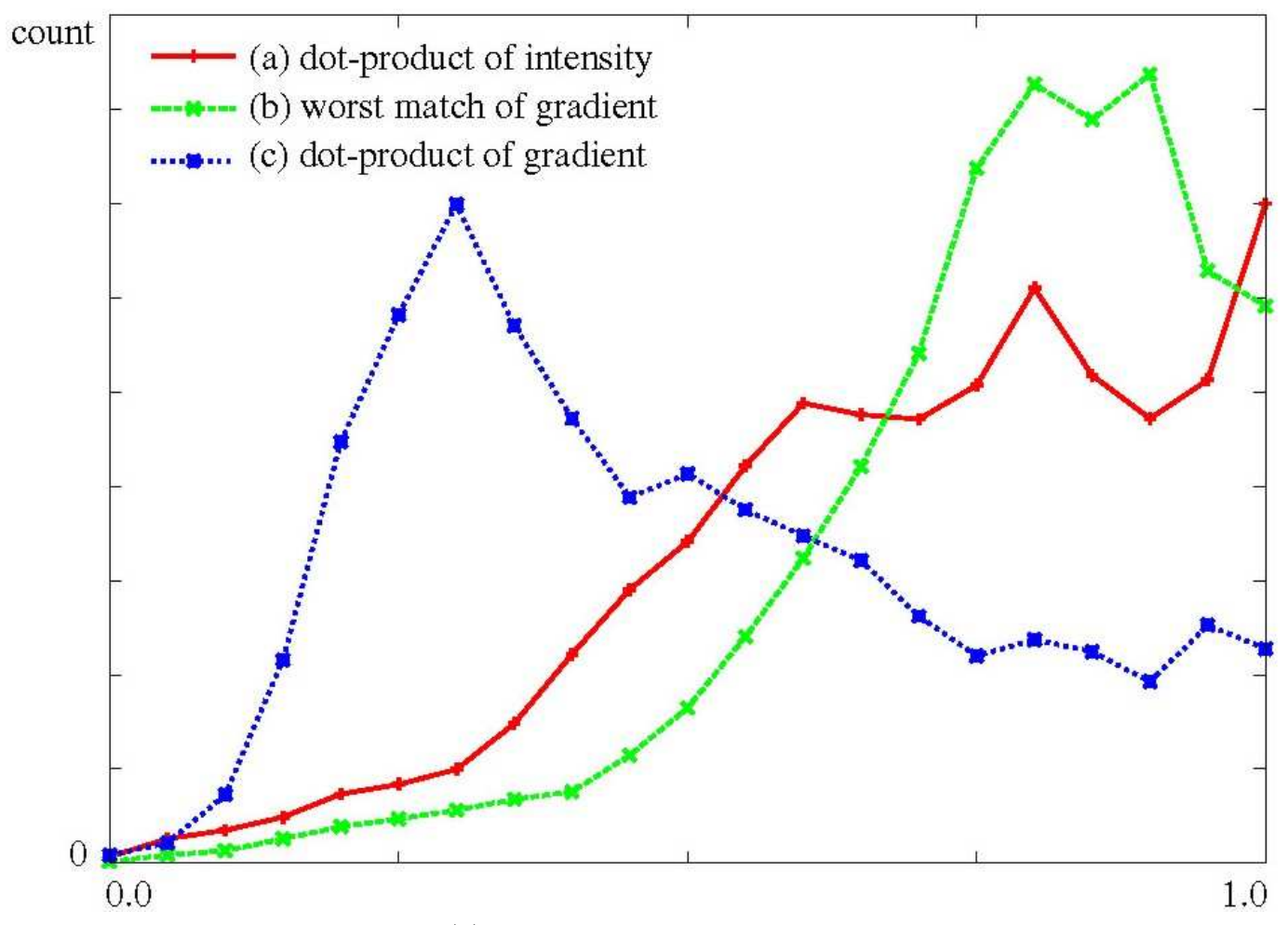

(d) histograms of function values

Fig. 3 Confusion matrices of correlation functions: The correlations between all pairs of dithering patterns of a DLP projector are computed using three distance functions: (a) the dot-product of normalized intensities, (b) the worst match of gradient components (similar in spirit to the function proposed by Zhang et al. [48]), and (c) the dot-product of normalized gradients. The axes of each figure denote the 8-bit gray values input to the projector. The correlation is normalized into 0.0-1.0 for comparison and encoded in color for illustration. Figure (d) shows the distributions of the correlation. The dot-product of normalized gradients yields low correlation when two vectors differ, and therefore is suitable to determine the identify of two vectors from the correlation.

patterns used to find correspondences between the camera and projector. The patterns can be classified into three categories according to their coding strategy. The first class of techniques uses time-multiplexing to encode the temporal location of the projector pixels and group the pixels over time. Sato and Inokuchi [36] proposed the shape acquisition system that uses binary illumination patterns. To achieve more efficiency and robustness a variety of improved patterns, such as gray codes [2], $n$ ary codes [12], or stripe boundary codes [10], have been proposed. The second class of the techniques, neigh- borhood codification, encodes information into a single image using spatial coherence. Boyer and Kak [3] proposed a color coding of adjacent stripes to identify the projector pixels. Zhang et al. [48] and Morita et al. [23] used respectively $1 \mathrm{D}$ and $2 \mathrm{D}$ color stripes defined by the pseudo random sequences that guarantees the uniqueness of local adjacency. The third class of techniques are direct codification where the locations of projector pixels are encoded into gray-level or color values of a single image [21]. There are also combinations of these three coding strategies to improve the speed, accuracy, 


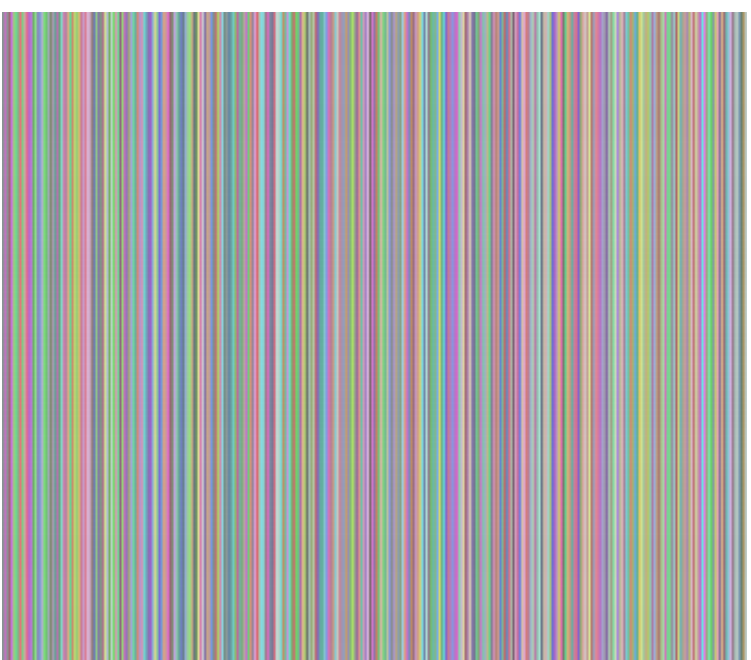

(a) Illumination pattern

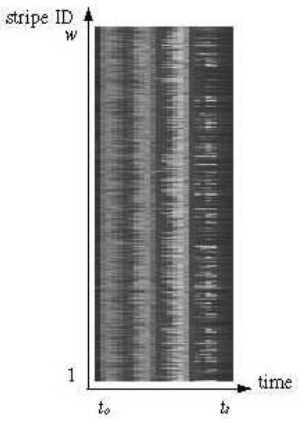

(b) Temporal dithering

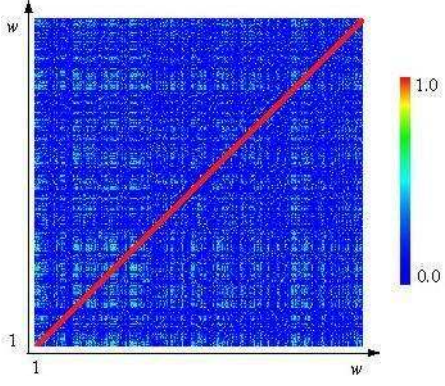

(c) Confusion matrix
Fig. 4 The illumination pattern for 3D shape acquisition. (a) We generated a color stripe pattern composed of size 512 distinct colors $(w=512)$. (b) The temporal dithering patterns of a DLP projector for the colors used in the stripes. (c) The confusion matrix of the dithering patterns, showing high correlation only along the diagonal.

and robustness of $3 \mathrm{D}$ reconstruction. Refer to [35] for a comprehensive survey.

In theory, any of these illumination patterns can be used for high-speed shape acquisition. The existing algorithms of 3D shape reconstruction can be applied directly to high-speed systems. In practice, however, generating arbitrary illumination patterns requires a DMD kit, which is prohibitively expensive. One possible way to overcome the limitation is to modify the projector hardware to generate arbitrary binary patterns at high speed $[20,14]$. Instead, we propose to use the dithering patterns from DLP projectors.

The dithering function transforms the 24-bit RGB color input, sent to the projector, into a binary sequence. It is then converted into a sequence of grayvalued intensities due to the mismatch of the synchronization between camera and projector, and the inten-

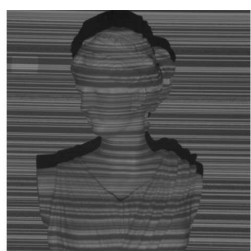

Frame 5555

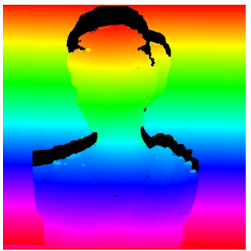

(b) Stripe correspondence

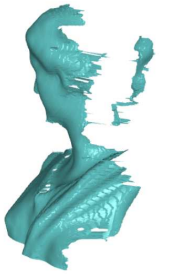

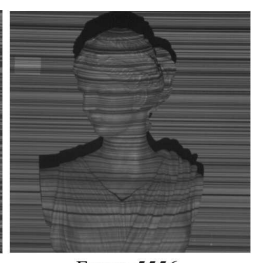

Frame 5556 (a) Input frames (3 of 25)

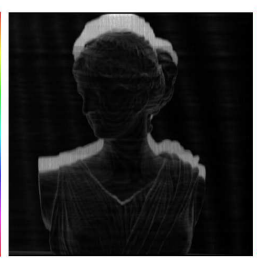

(c) Matching error

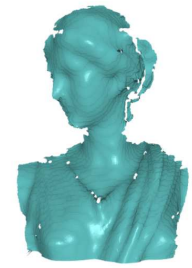

(e) Recovered structure

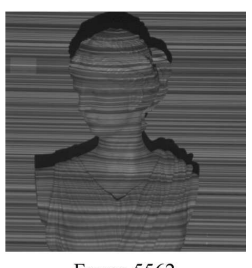

Frame 5562

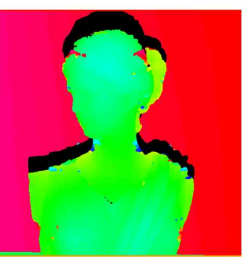

(d) Disparity map

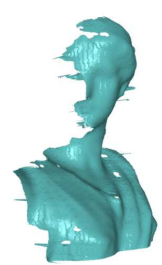

Fig. 5 Results of 3D reconstruction using the DLP projector for a static venus bust: (a) Three frames captured by the high speed camera illustrate the fast modulation of illumination incident on the scene. 20 continuous frames are used to match the intensity variation observed on the scene point against the normalized intensity variation observed on the vertical plane behind the object. (b) The best match finds correspondences between projector and camera pixels. The error map is shown in (c). The (d) disparity and (e) recovered shape of the object is shown from different viewpoints.

sity leakage between adjacent frames. Let $I(v, t)$ be the intensity of a scene point on a white plane, illuminated by a projector with the input intensity $v$, and observed by a high-speed camera at time frame $t$. The intensity vector $\mathbf{I}(v)=\left(I\left(v, t_{0}\right), \ldots I\left(v, t_{1}\right)\right)$ acquired for a projector-frame cycle $t=t_{0}, \ldots, t_{1}$ characterizes the image intensity at a scene point illuminated by a projector pixel with intensity $v$. Conversely, when we obtain an intensity vector at another scene point, the corresponding projector input $v^{\prime}$ can be identified by finding the maximal correlation between $\mathbf{I}(v)$ and $\mathbf{I}\left(v^{\prime}\right)$ for all $v$.

Ideally, the correlation between two vectors $v$ and $v^{\prime}$ would be maximum when $v=v^{\prime}$, and would become as low as possible when $v \neq v^{\prime}$. It is also desirable that a large number of pairs $\left(v, v^{\prime}\right)$ yields low correlations in order that each projector intensity in a stripe pattern be robustly distinguished. Since the optimal correlation function depends not only the dithering function of the projector, but also the performance of a high-speed camera, we have investigated three different functions: 
(a) Normalized dot-product of intensities:

$$
D_{a}\left(v_{1}, v_{2}\right)=\frac{\mathbf{I}\left(v_{1}\right) \cdot \mathbf{I}\left(v_{2}\right)}{\left\|\mathbf{I}\left(v_{1}\right)\right\|\left\|\mathbf{I}\left(v_{2}\right)\right\|}
$$

where $v_{1}$ and $v_{2}$ are intensities input to a projector.

(b) Worst match of image gradients :

$$
D_{b}\left(v_{1}, v_{2}\right)=\max _{t_{0}, \ldots, t_{1}-1}-G(v, t)
$$

where $G(v, t)=I(v, t+1)-I(v, t)$.

(c) Normalized dot-product of gradients:

$$
\begin{aligned}
& D_{c}\left(v_{1}, v_{2}\right)=\frac{\mathbf{G}\left(v_{1}\right) \cdot \mathbf{G}\left(v_{2}\right)}{\left\|\mathbf{G}\left(v_{1}\right)\right\|\left\|\mathbf{G}\left(v_{2}\right)\right\|} \\
& \text { where } \mathbf{G}(v)=\left(G\left(v, t_{0}\right), \ldots G\left(v, t_{1}-1\right)\right)
\end{aligned}
$$

The normalized dot-product of intensities is one of the simplest and commonly-used correlations of two vectors. It, however, has high correlation values for a large number of pairs as illustrated in Figure 3 (a), which implies potential ambiguity in correspondence at these pair of intensities. The worst match of gradient components is similar in spirit to the correlation function used by Zhang et al. [48] It penalizes the disagreement of sign in gradient components, and takes a large value only when all components have consistent signs. However, as shown in Figure 3 (b), this function also has considerable ambiguity because the camera and projectors are not perfectly synchronized and the high-speed camera suffers from the noise due to the temporal blurring between frames. The effect of temporal blurring can be seen in Figure 1 (d). The observed image intensities have continuous distribution whereas the output of a DLP projector is originally binary. Accordingly, we decided to use the normalized correlation of gradients to compute the correlation between the observed intensity vectors (Figure 3 (c)). Figure 3 (d) shows the distributions of the three correlations. The dot-product of normalized gradients yields low correlation when two vectors differ, and therefore is suitable to determine the identify of two vectors from the correlation.

Note that we computed the correlation only between 8-bit gray values, while ideally all pairs of 24-bit RGB colors should be compared. We simplified the experiment based on the following consideration: The temporal dithering pattern in each color channel looks similar to that of gray values. The global optimality is not required either; In fact, we used the normalized correlation of intensity in our earlier work [24].

The illumination pattern is then created by choosing the projector intensities that can be robustly distinguished by the correlation function $D_{G}$. Since we do not have the confusion matrix of full 24-bit RGB colors, the stripe pattern is generated by randomly choosing distinct RGB colors such that the correlation of any pair of the colors is less than a certain small number. Figure 4 shows the illumination pattern we used in our experiments. The number of the stripes in the pattern is $w=1024$, but is reduced to 256 in the figure for visualization purpose. Each RGB color $v=(r, g, b)$ of the stripe is generated such that $r, g, b \in[100,255]$ because dark illumination leads to low signal-to-noise ratio of camera images and causes unreliable decoding.

\subsection{Reconstruction Algorithm}

Given the high-speed images of an object illuminated by a DLP projector, the correspondence from camera pixels to projector pixels can be determined by computing the correlation functions described in Section 3.1. It would seem that simply knowing both the slide sent to the projector and the ditherings from Figure 1 would be sufficient for finding camera-projector correspondences. However, this method does not result in correct correspondences since the camera and projector are not synchronized. Finding the exact phase difference between the camera and projector would result in a large search problem, which we wish to avoid. How do we synchronize the frames from the projector and the camera? One approach is to include a small, diffuse planar patch in the scene where correspondence between the corners of the patch can be easily established (say, manually). This correspondence allows us to synchronize the measured intensity vector with the temporal dithering.

\subsection{Results}

Consider a high speed camera viewing a dynamic scene that is illuminated by a DLP projector. We use a single image composed of $1 \mathrm{D}$ patterns as the input to the projector. The correspondences from camera pixels to the stripe are sought using the epipolar constraints of the camera and projector.

We performed several experiments with both static dynamic objects: a static statue, a waving cloth, a grasping hand, a face, and a hand spinning a pen. A homogeneous vertical plane is used as the background behind the scene. The dithering $D_{I}(t)$ can be captured from the pixels on this plane and simultaneously with the object. Hence, in this setting, we simply correlate the normalized brightness vector $I(t)$ of a point on the object with the dithering vector $D_{I}(t)$ at every pixel $C$ on the background plane and no additional synchronization is needed.

Here, twenty frames were used to obtain correspondences, taking $20 / 3000=1 / 150 s$. In general, the num- 

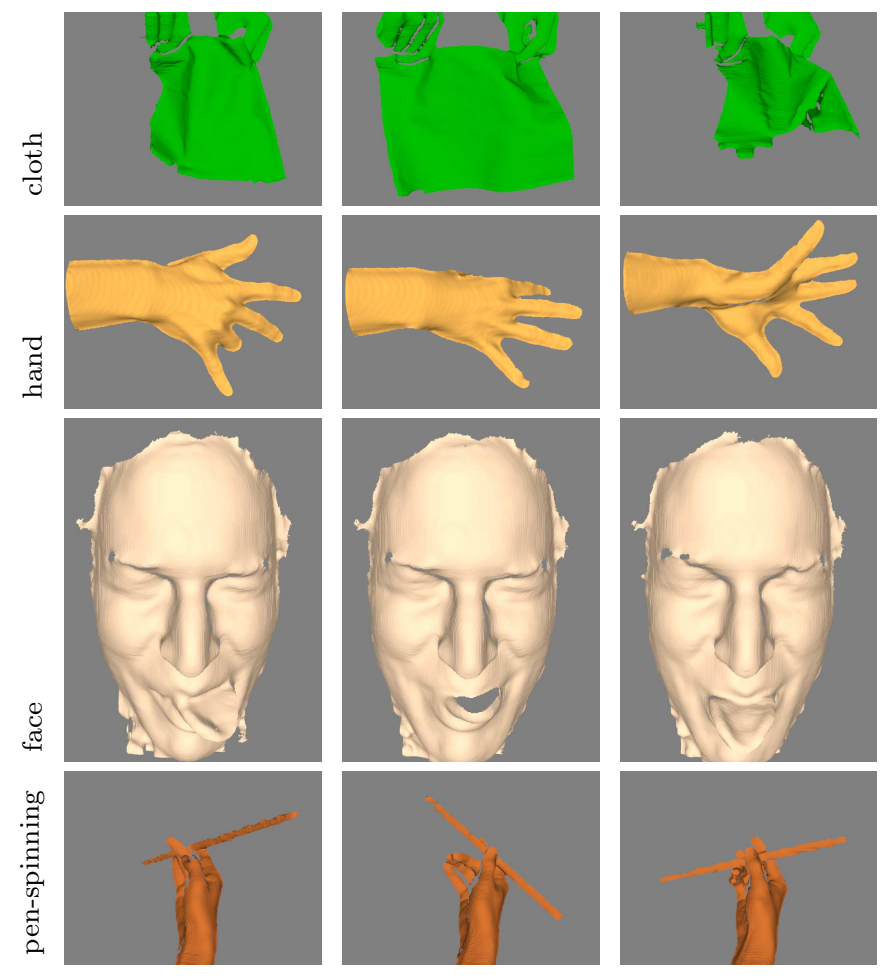
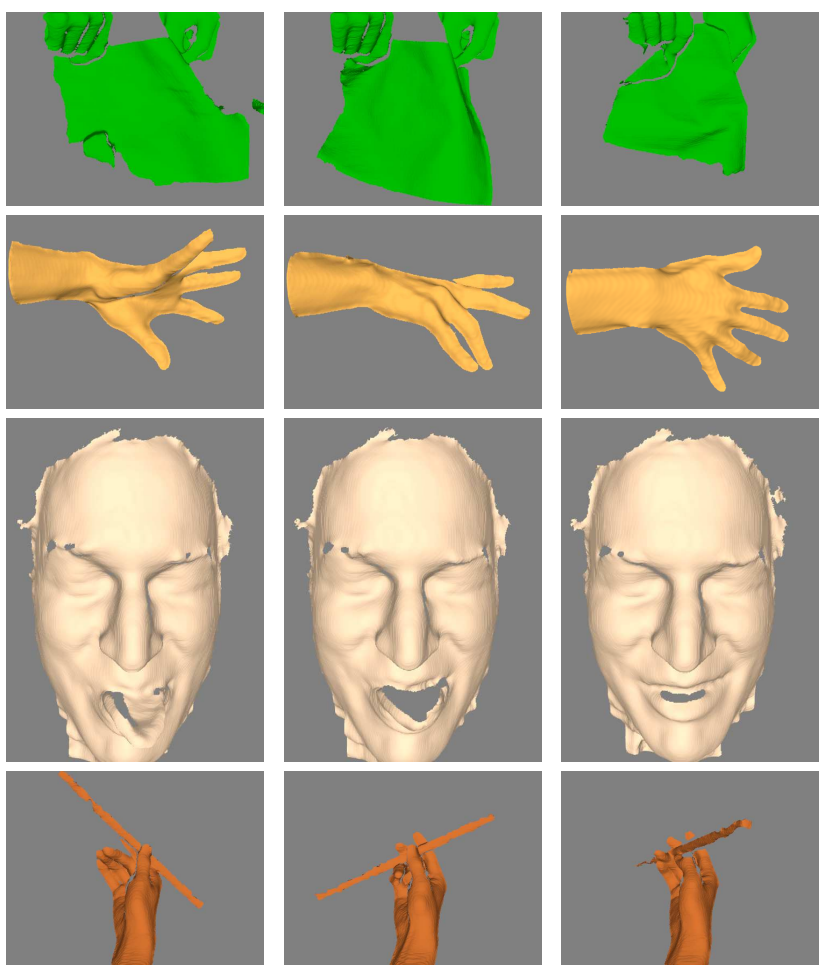

Fig. 6 Results of 3D reconstruction using the DLP projector for dynamic objects: a waving cloth, a grasping hand, a face, and a hand spinning a pen. Each depth map is reconstructed using successive 25 frames in the high-speed images captured at $3000 \mathrm{~Hz}$.

ber of frames necessary depends on the desired matching accuracy and the SNR of the acquired images. By sliding the 20 frame window across the full image sequence, $3 \mathrm{D}$ reconstruction can be obtained at rates as high as $3 \mathrm{kHz}$ (speed of camera). However, in practice, the rate of reconstruction is lower considering the speed of the object's motion.

Figure 5 shows the correspondences, the disparity and the reconstructions obtained for a venus bust. Any holes are only due to stereo shadowing. This example, although static, was chosen to highlight the quality of our stripe correspondences, which match (with low error) the horizontal lines of the pattern, as shown in Figure 5 (b). Figure 6 shows the reconstructed shapes of dynamic objects. In the top row we show a flapping cloth, and note that we are able to reconstruct very fast moving features such as folds and creases. The second row shows fast hand-motions, where we have been able to capture the muscle deformations and stretching of the fingers and palm. In the third row we show a human 'making faces' and sticking out his tongue. Again, facial features whose detection requires fast reconstruction, such as fine wrinkles, are clearly recovered. In the last row we show a hand spinning a pen. This is the most difficult case, since the pen is a long thin structure and its edge moves at almost the camera frame rate. Still, our matching is robust and we are able to reconstruct the scene reasonably well.

Camera frame-rates: We note that the dithering can also be observed at lower frame rates and hence a lower cost camera may be used for slower reconstructions. We repeated our experiments for the same scene at four lower frame rates $-1500 \mathrm{~Hz}, 600 \mathrm{~Hz}, 300 \mathrm{~Hz}$ and $120 \mathrm{~Hz}$. Figure 7 shows the reconstructions obtained. The frame rate of $120 \mathrm{~Hz}$ is too low to capture the required intensity variation and hence, the projector-camera pixel correspondences are unreliable. However, at $500 \mathrm{~Hz}$, the reconstruction quality is still acceptable indicating that the temporal dithering can be exploited even at this frame rate.

\section{Illumination De-multiplexing for Appearance Capture}

Acquiring scene appearance from multiple lighting directions is necessary for image-based relighting and photometric recovery of scene properties (BRDF and 3D structure). In most works, the scene is assumed to be static and the acquisition can take any where from a few seconds to several minutes. Using DLP illumination, we capture the appearances of a dynamic scene from multiple lighting directions. For this, we draw upon the idea of illumination de-multiplexing [38], where the images 


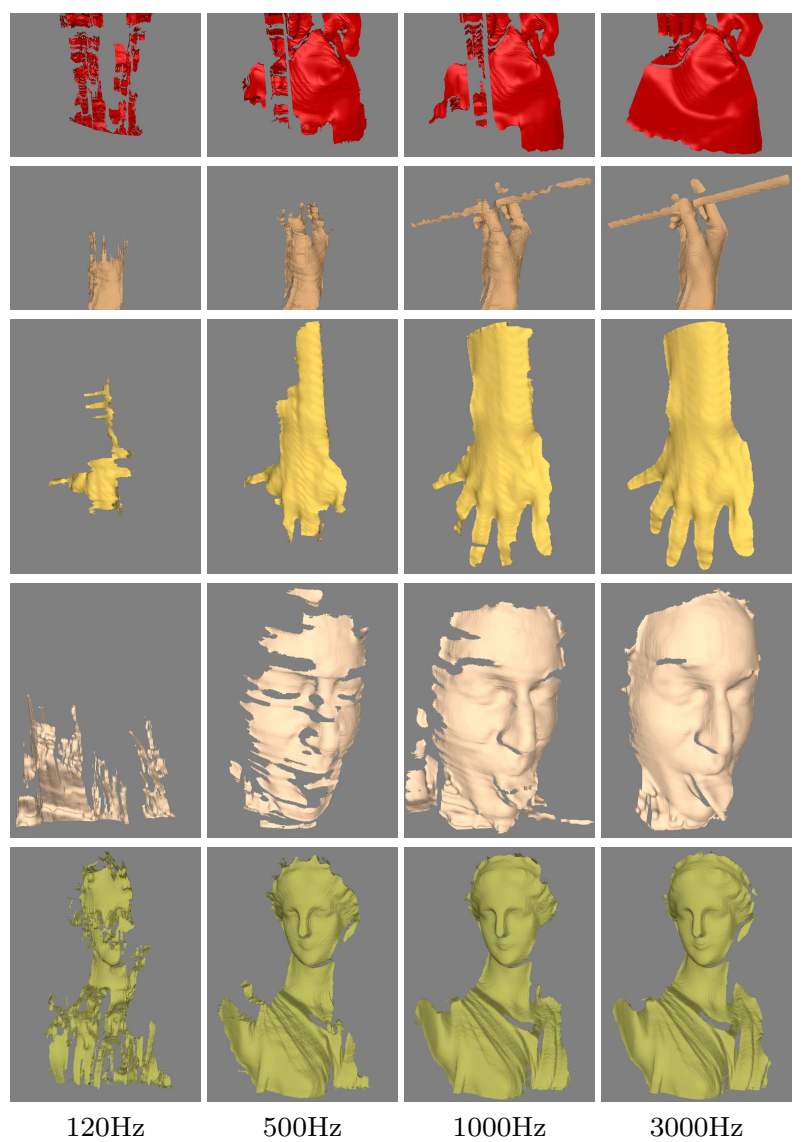

Fig. 7 Reconstructions obtained using videos captured at reduced frame rates. Even at $500 \mathrm{~Hz}$, the quality of the reconstruction obtained remains acceptable indicating that temporal dithering can be exploited at this frame rate.

of the scene are simultaneously captured from multiple source directions and de-multiplexed in software to obtain the desired images under each lighting direction. This technique increases the signal-to-noise of the captured images while keeping the number of captured images unchanged.

The difference between Schechner et al. [38] and our technique is in the coding: they use binary Hadamard codes, whereas we rely on the temporal dithering of DLP illumination. The acquisition setup consists of three DLP projectors (Infocus IN38 and LP120, and Sony XGA DataProjector) that simultaneously illuminate the scene from different directions. Since we wish to illuminate the scene uniformly, a single constant brightness image is input to each of the projectors.

The three projectors differ in their brightness and contrast ratings and dithering behaviors. The captured intensity at time instant $t$ is written as a sum of ir- radiances due to the illuminations from all projectors $(k=1 \ldots 3)$ :

$I(t)=\sum_{k=1}^{3} D_{k}(t) E_{k}(t)$

where, $D_{k}(t)$ is the dithering intensity of the projector $k$ at time $t$ and $E_{k}(t)$ is the irradiance due to the scene as if illuminated only from projector $k$ but with unit intensity. The intensities $D_{k}(t)$ can be obtained by observing a calibration object; this could either be a stationary mirror/lambertian sphere placed in the scene, or a diffuse lambertian background plane placed such that the edges of the three projector's FOV are visible. The observed irradiances $I(t)$ over time form a linear system which is solved to obtain the appearances $E_{k}(t)$ of the scene from each individual lighting direction. In practice, since the projectors are not synchronized when they illuminate the scene, the dithering intensities $D_{k}$ vary significantly over time, and hence the linear system is well-conditioned.

Figure 8 shows the results of applying the above approach to a scene with a falling wiry ball. Notice the 3 shadows of the ball and the mirror sphere that appear mixed in the multiplexed image $I(t)$. For robustness, we use 10 frames to solve the above linear system. Notice separation of the shadows in the demultiplexed images. As before, the effective rate of demultiplexing depends on the SNR in the high speed camera. We have thus far ignored color information, however, when the three DLP projectors emit intensities in different spectral bands, the de-multiplexing algorithm can be used to colorize the acquired high speed gray-scale video.

\subsection{Colorizing High-speed Video by Demultiplexing}

In the previous section we showed how choosing the right projector intensities can enable fast illumination demultiplexing for dynamic scenes. In this section, we show one application of demultiplexing to colorize a high speed video. In Figure 9 we show our setup in (a), where three DLP projectors are used to illuminate a scene and their centers of projection are kept close to minimize scene shadows. Instead of a reflective sphere, we use a diffuse background to estimate the intensities, as explained in the next section. Each projector is fitted with a color filter, and the demultiplexed images obtained are the response of the scene to red, green and blue illumination. We can, therefore, create a color image of the scene as shown in the figure. Since we require 30 images for the demultiplexing, and the frame rate of the camera was $3000 \mathrm{hz}$, the effective frame rate of the color video is $100 \mathrm{hz}$. 


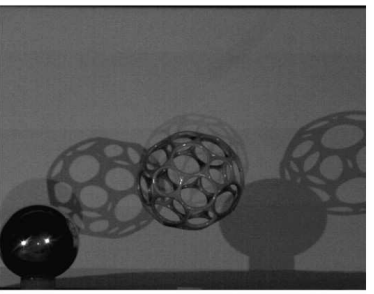

Frame 969
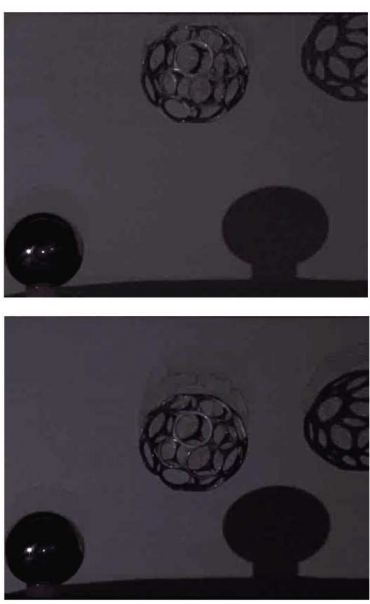

Images illuminated by projector 1

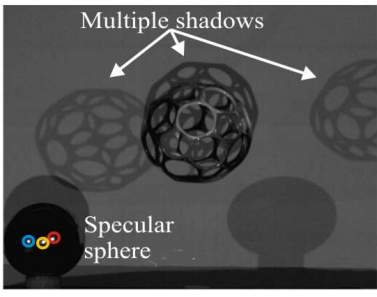

Frame 457

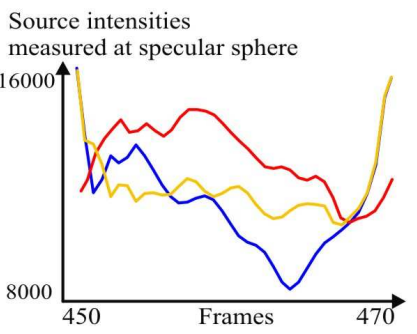

(a) Multiplexed Illumination
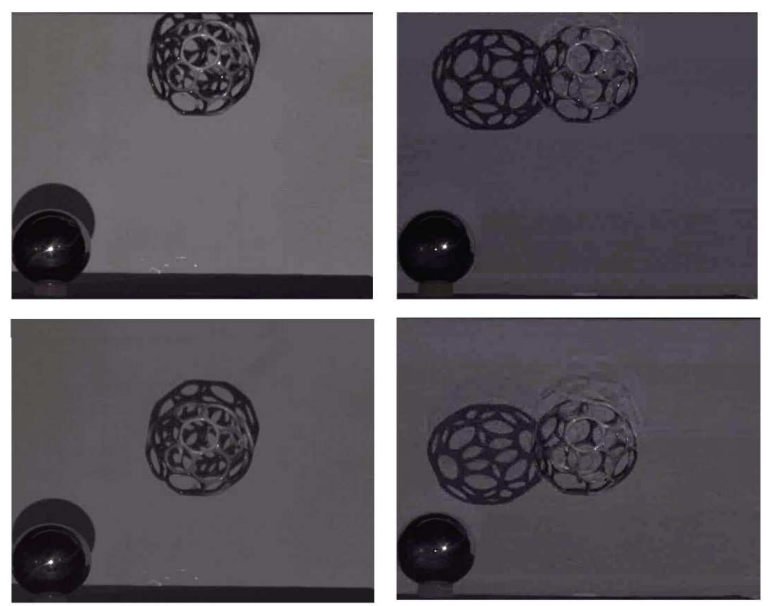

Images illuminated by projector 2

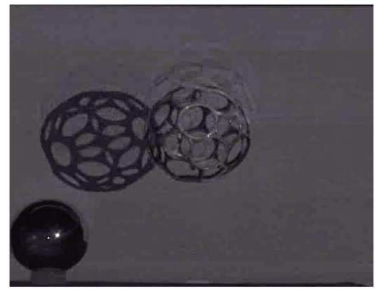

Images illuminated by projector 3

(b) De-multiplexed Illumination

Fig. 8 Demultiplexing illumination from three projectors to create appearances under each lighting direction: The scene consists of a wiry polyhedral ball falling vertically. Notice the mixing of the shadows in the multiplexed images in (a). A mirror sphere is placed in the scene to measure the dithering intensities $D_{k}$ from the three projectors. (b) The results of demultiplexing for two frames in the video sequence. A total of 10 frames are used to demultiplex. Some ghosting artifacts are due to the noisy estimation of source intensities $D_{k}$. The variation in the contrast and brightness of the three demultiplexed images are due to the quality of the three projectors. Projector 2 (InFocus IN38) is the most recent one and has the highest quality.

Our Photron PCI-1024 camera produces black and white images, while a similar color high speed would cost almost double this. Therefore we believe our method is extremely practical and useful. One issue of our approach is that commercial DLP cameras have a color wheel inside them, that have red, green, blue and white filters turning at about $120 \mathrm{hz}$. This violates our assumption that each projector corresponds to only one component of the spectrum. Therefore, we have removed the color-wheel in the DLP projectors before performing the experiment. In the last row of Figure 9 we show one colored frame, but please view the video on the website ([18]) for a full length clip.

\section{Illumination Multiplexing for Photometric Stereo}

Photometric stereo is a widely used method to recover the surface normals and albedos of objects that are pho- tographed under different lighting directions. There are many variants of this approach and we chose the classic algorithm by Woodham [45] for its simplicity. In that work, the appearance of the scene under three independent distant illumination directions is used to obtain the surface normal of the scene point. We will extend this approach for fast moving scenes that are simultaneously illuminated from different directions.

The scene in our experiments consists of a mirror sphere, a white diffuse background plane and a fluttering white cloth flag (Figure 10). These are illuminated by three DLP projectors simultaneously from different directions and viewed by a high speed camera recording images at $3 \mathrm{kHz}$. Since each projector must uniformly illuminate the scene, we provide a single constant brightness image as input to each projector (with different brightness values). The projectors are de-synchronized and hence, the "multiplexed illumination" results in significant variation in the observed intensities. In ad- 


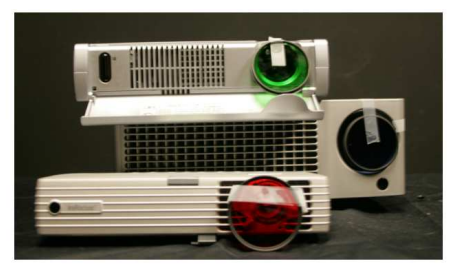

(a) DLP Projectors with color filters

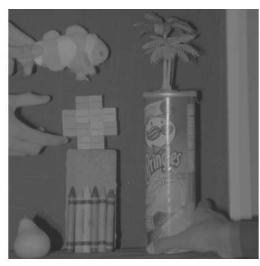

(b) Input images of a dynamic scene at $3000 \mathrm{hz}$

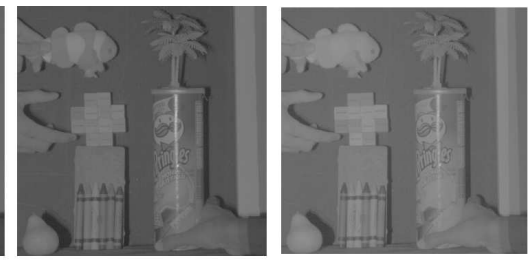

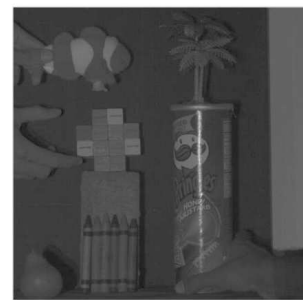

Red

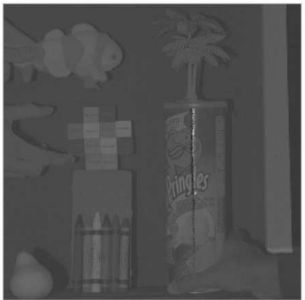

Blue

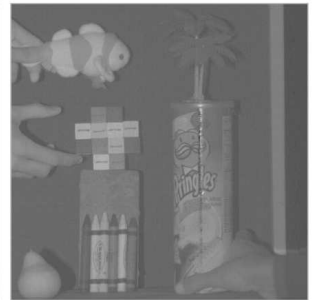

Green

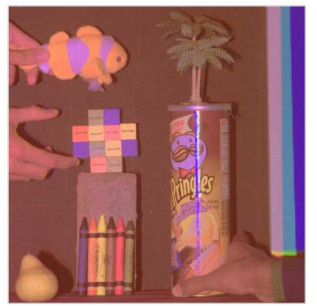

(d) Color Image

(c) Demultiplexed Images

Fig. 9 Colorizing a high-speed video: Here we apply the demultiplexing technique, where red, green and blue color filters have been applied to the projectors. The projector color-wheels were removed before this experiment was run. The input images show the white cardboard at the right of the scene, on which the edges of the three projected slides are visible. These three edges are clearly demarcated in the demultiplexed images. The intensities of these demultiplexed images are the responses to the red, green and blue components of the scene albedos (up to a scaling factor). These are concatenated to produce a color high-speed video. Note that while off-the-shelf DLP projectors are available on the order of 100s of dollars, a color high speed camera would price an order of magnitude higher.

dition, the projectors and camera are far enough away from the scene to assume orthographic viewing and distant lighting.

Each projector has a different field-of-view (FOV) and therefore (in Figure 10) different parts of the background plane are illuminated by different projectors. This allows the edges of each projector FOV to be perceived, as can be seen in the figure. The patches around these edges are illuminated by different pairs from the three individual projectors. We use the normalized intensities of these patches as estimations of combinations of projector intensities and demultiplex the scene. We then apply classical lambertian photometric stereo to the demultiplexed video to obtain the surface normal, using a mirror sphere that provides the projector directions. A window of length 10 frames achieved robust results. A sliding window of 10 frames can be used to generate the normals up to a rate of $3 \mathrm{kHz}$. As before, the speed of the object determines the effective performance rate. Figure 10 shows the reconstructed shape from the surface normals. Note the creases and folds in the flag that are faithfully reproduced.

\section{Flutter Flash: Motion-blur under DLP Illumination}

Motion-blur occurs when the scene moves more than a pixel within the integration time of a camera. The blur is computed as the convolution of the scene mo- tion with a box filter of width equal to the camera integration time. Thus, images captured of fast moving objects cause a smear across the pixels losing significant high frequencies. Deblurring images is a challenging task that many works have addressed with limited success. A recent approach by Raskar et al. [32] uses an electronically controlled shutter in front of the camera to modulate the incoming irradiance at speeds far greater than the motion of the object. In other words, the box filter is replaced by a series of short pulses of different widths. The new convolution between the object motion and the series of short pulses results in images that preserve more high frequencies as compared to the box filter. This "Flutter Shutter" approach helps in making the problem better conditioned. Our approach is similar in spirit to [32] with one difference: the fast shutter is simulated by the temporal dithering of the DLP illumination. Note that the DLP illumination dithering is significantly faster than mechanical shutters ${ }^{1}$.

In Figure 11(a)-(c), we show images taken under DLP, skylight and fluorescent illumination. The object is a cardboard sheet translating from left to right with the PSF approximated by a small white dot placed on the sheet. We use this as a good starting point for blind deconvolution methods. For skylight and fluorescent light, we also tried the 'box' PSF which assumes

1 Faster shutters can be realized by electronically triggering the camera. 


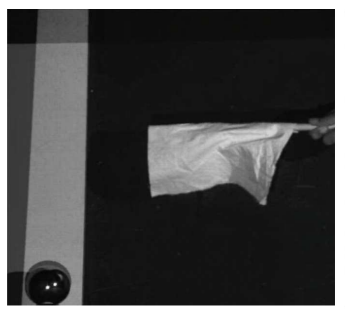

(I) Three consecutive frames under unsynchronized DLP illumination
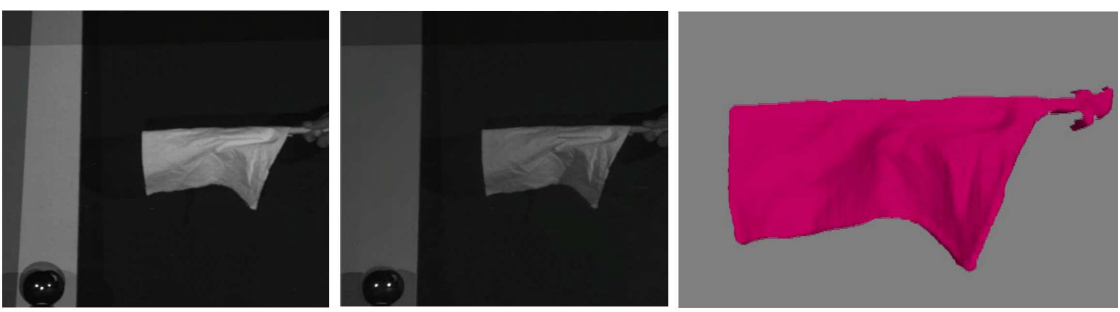

(II) Reconstructed shape

Fig. 10 Photometric stereo: The scene consists of a fast moving fluttering cloth flag, a background diffuse plane and a mirror sphere. Three DLP projectors simultaneously illuminate the scene and the camera operates at $3000 \mathrm{~Hz}$. The projectors and camera are far enough away from the scene to assume orthographic viewing and distant lighting. The patches on the calibration plane correspond to combinations of different pairs of the three projectors. We use these to demultiplex the scene and calculate the surface normals from the demultiplexed frames by classical photometric stereo. A window length of 10 frames achieved robust results.

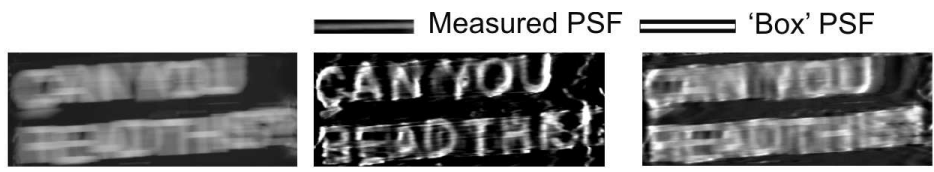

Motion blur

Deblurred image

(a) Skylight illumination
Deblurred image

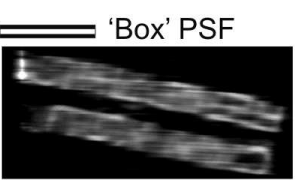

Deblurred image

(b) Fluorescent illumination

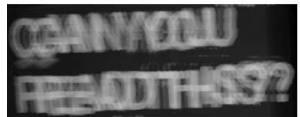

Motion blur

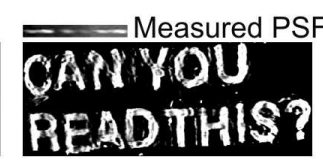

Deblurred image

(c) DLP illumination

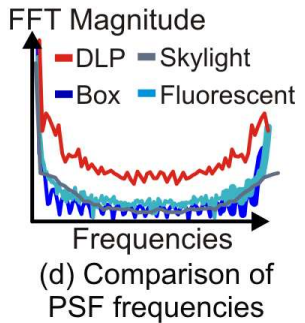

Fig. 11 DLP photographs contain higher frequencies compared to other types of illumination: In (a) and (b) we show deconvolution results with skylight and fluorescent illumination. The blind deconvolution algorithm was given the intensity profile of a white dot as a starting point. The result for deblurring the same motion under DLP illumination (c) can be read easily. Analysis of frequencies in the recovered PSF shows DLP illumination preserves high frequency information.

constant incident illumination during exposure. Note that the best deconvolution occurs with the DLP photograph. In Figure 11(d) we show the frequencies of the PSFs. Note that the highest frequencies are due to the DLP illumination. Although previous work has either used camera apertures to create similar images ([32]) or shown some deblurring results $([24])$, we are the first to analyze and compare the frequencies of DLP illumination to other types of lighting.

Deblurring images using flutter-flash (or, as in [32], flutter-shutter) makes some assumptions about the scene for example, we require uniform motion of a rigid body. For scenes with articulated and deformable objects, a global flutter does not help to estimate the blurring function. This is because the blur kernel is local and can be different at each pixel, making the deblurring problem hard. Instead of tackling this tough problem, in this section, we take a alternative approach. We will demonstrate how to present the high-frequency motion information available in the DLP photographs, without deblurring them. This approach is inspired from computational photography techniques that complement deblurring methods.

\subsection{The DMD-Colorwheel Effect}

Projecting color images involves synchronized control between the DMD chip, which has a frequency of a $10^{6} \mathrm{~Hz}$, and the color wheel, which rotates at $120 \mathrm{~Hz}$ and 
is divided into red, green and blue filters. This 'rainbow effect' is well-known to display researchers who wish to remove or reduce it $([29],[15],[30],[41],[13],[28],[22])$. Many researchers even remove the color wheel to increase the projector contrast in their experiments ([19], [4]). Instead of treating this effect as a problem that must be compensated for, the DLP illumination can be exploited to photograph dynamic scenes. Therefore DLP illumination can produce strobing effects for both real-time and high-speed scenes, and we term this the DMD-Colorwheel effect.

This strobing effect is special for two reasons. First, at high-speeds (the DMD part of the effect), the dithering is due to binary switching of a MEMS mirror. Therefore, the illumination changes sharply, almost as a delta or step function. This is in contrast to the closest competitor in illumination devices, which are high-speed LEDs. These solid state devices have a 'ramp-up' time, and would cause blurring in strobe-photography of the very fast events that we capture, such as an air-balloon bursting or slingshots. In addition these devices are more expensive that off-the-shelf DLP projectors. Secondly, at real-time speeds (the colorwheel part of the effect), the strobe effect creates multiple colored copies of the object. This allows interesting color demultiplexing effects that can illustrate the motion. These are difficult to perceive as images, and so we only include them as videos on our DLP photography website, [17]. Instead, in this section, we will focus on motion illustrations that can be displayed as still photographs. We call images of scenes under DLP illumination taken by still or low-frame rate cameras, as DLP photographs.

\subsection{DLP Photography}

The DMD chip has a frequency of a $10^{6} \mathrm{~Hz}$, but the dithering in a commercial projector occurs at around $f=10000 \mathrm{~Hz}$ ([24]). This allow us to capture very high-frequency events such as the air balloon bursting as showing in Figure 12. Note that most other forms of illumination would cause blurring in this image. As an example, we show that under fluorescent lighting, viewed at $1000 \mathrm{fps}$, the balloon is smeared in a single frame, and this high-speed event is lost. However, when viewed under DLP illumination, the images at 1000fps show copies of the edge of the balloon. We used an Infocus In38 projector projecting a plain gray image of intensity 192 of 3000 lumens, viewed by a Photron PCI-1024 high-speed camera. Our setup enables photography of an event occurring at 10 times the frame rate of the viewing camera. Finally in the figure, we use three projectors that are unsynchronized creating a higher strobing frequency and therefore obtaining a similar photograph at a lower frame rate of $500 \mathrm{~Hz}$.

In contrast to the very high-speed events described above, the color component of the DMD-colorwheel effect is clearly visible for scenes with 'real-time' movements such as fast human motion. In Figure 13 we show pictures of a tabla (hand drum) being played, as well as a ballet dancer performing. Note that the copies appear at different colors, since they are illuminated when the color wheel turns the red, green or blue filters. We also show an image of a water-balloon bursting. The specularities of the water droplets are clearly colored red, green and blue, and these would have been blurred under fluorescent illumination.

\subsection{Summarizing Fast Events}

An image summarizing a video sequence can be created by stitching important frames together, as in shapetime photography $([9])$. However, the object must move slowly since otherwise motion blur will render the final result difficult to interpret. DLP photographs already summarize a short burst of action, since they contain multiple copies of moving objects. Applying a similar method as shape-time photography to a collection of DLP photographs creates a summary image for fast motion.

In Figure 14 we show images created by processing a volume of DLP photographs of a tabla player and a ballet dancer. We first separate the images approximately into the strobed and non-strobed part using a well-known intensity-based segmentation method ([46]). Except for the first image, the rest of the images are strobed. The top image is created by taking the intensity maxima of each pixel over all the photographs, which produces the effect of combining the different copies and gives a summary of the motions that occurred. In contrast, the bottom of the figure is creating by masking the high intensity portions of each image and pasting them on top of each other. Instead of blending the outputs, these summaries enforce an order into the images.

While the previous examples showed images taken at 'real-time' rates (such as a human dancing or moving) with the colorwheel effect, now we look at strobing created by fast moving scenes with the DMD chip. In Figure 15 we show image summaries of a slingshot shooting a rock at high speeds. This summaries were created by applying the method of [9] to high-speed frames. The first result taken under fluorescent illumination with a $1000 \mathrm{~Hz}$ camera, clearly shows highfrequency information. The same experiment taken with a $250 \mathrm{~Hz}$ camera shows motion blur. However, replacing 


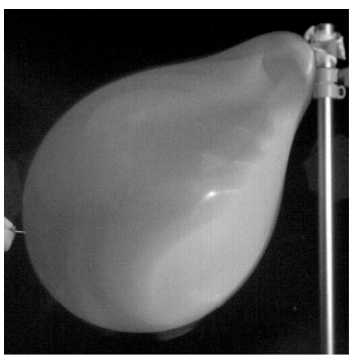

Fluorescent illumination at $3000 \mathrm{hz}$

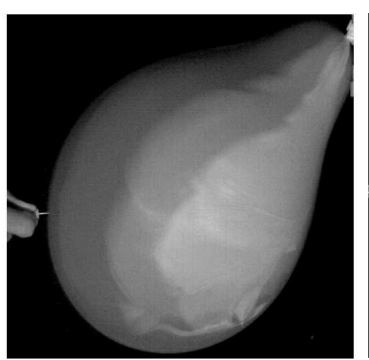

Single DLP projector with $3000 \mathrm{hz}$ camera
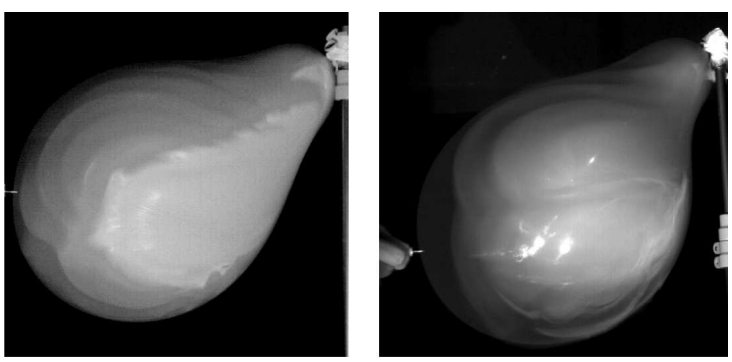

Three DLP projectors at $500 \mathrm{hz}$

Fig. 12 DLP photographs of a bursting balloon: An air balloon bursting can be captured fully using a 10000hz camera. In the first image we show what happens when the event is captured under fluorescent illumination, with a lesser rate of $3000 \mathrm{hz}$. In one frame the event is lost in motion blur. In the next two images, we show images taken under 3000hz, but this time with DLP illumination. Notice the multiple copies of edge of balloon as it moves. We are able to capture images of this high-speed event, due to the temporal dithering of the DMD device in the projector. Similarly in the last image, we use three DLP projectors, which are not synchronized. We are able to capture a balloon bursting with a $500 \mathrm{hz}$ camera, which is much slower than the speed of this fast event.
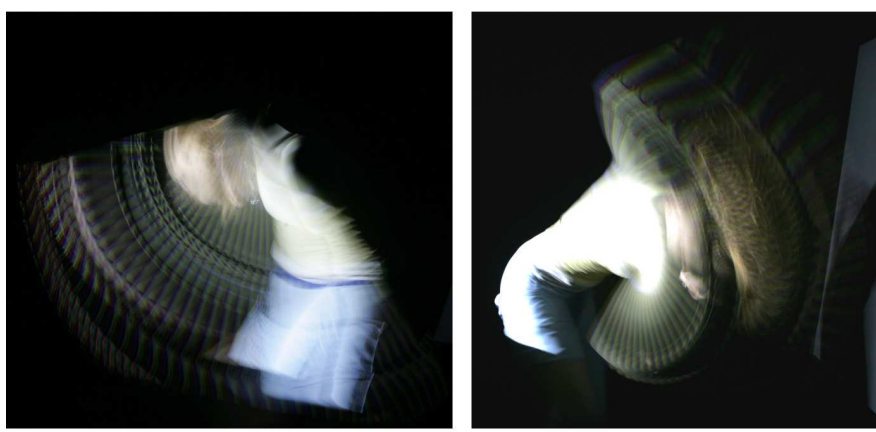

Three poses of a ballet dancer

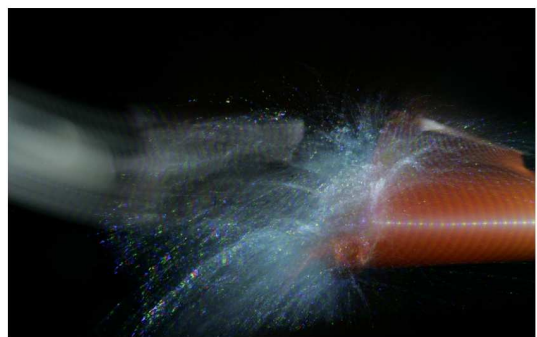

Water-balloon burst

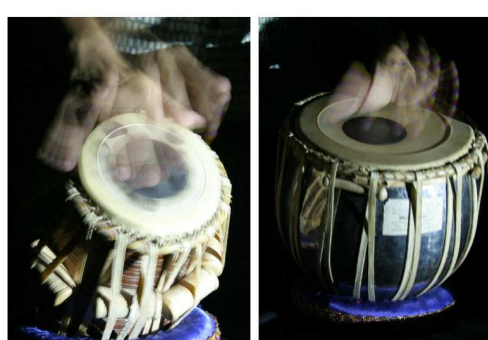

Close-ups of tabla drumming
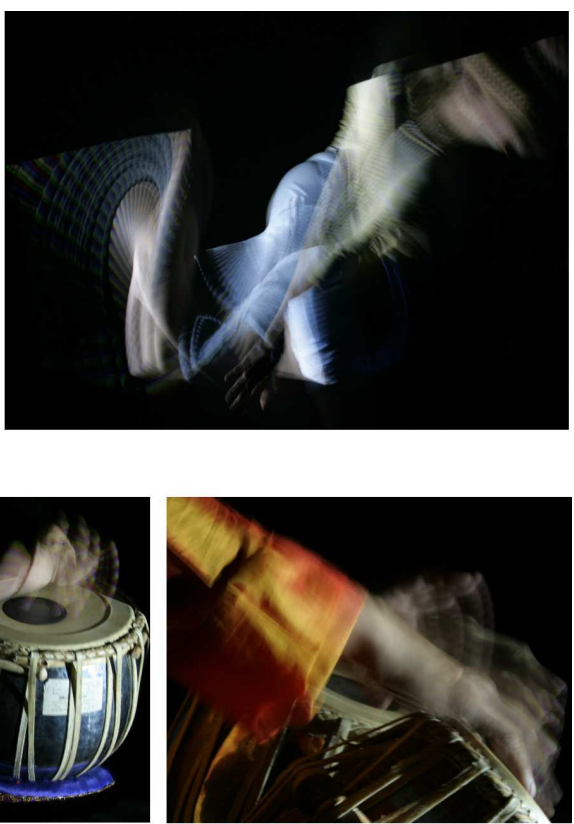

Side-view of tabla drumming

Fig. 13 Selected DLP photographs: We photographed two artists, a ballet dancer and a tabla (hand drum) player, under DLP illumination. Both activities are 'real time' and the color wheel effect dominates the images. The camera exposure was 1 second. We also show a balloon bursting, where the specularities of the water droplets are clearly colored red, green and blue. These would have been blurred under fluorescent illumination.

the fluorescent light with a DLP projector, creates an image on the right with the same high-frequency information obtained by a $1000 \mathrm{~Hz}$, but done with a $250 \mathrm{~Hz}$ camera. Therefore, fast strobing of a DLP projector can enhance the effective frame rate of a camera by allowing to capture scenes moving faster than its frame rate. In the second row, we show a more interesting scene involving a collision of a slingshot rock and a stationary plastic pear. By applying [9]'s method to different subsets of the video, we can summarize different aspects of the event.

\section{Temporal Dithering Characteristics: A Case Study}

In the previous sections, we have shown how the temporal dithering of a DLP projector can be used to extend any active vision technique to dynamic scenes. In all our experiments, we used patterns that were selected heuristically to provide best results. Providing a more theoretical analysis is difficult, since, in our experience, the dithering codes drastically differ with small changes in projector controls (such as contrast or brightness) and environment (such as temperature) as well as be- 


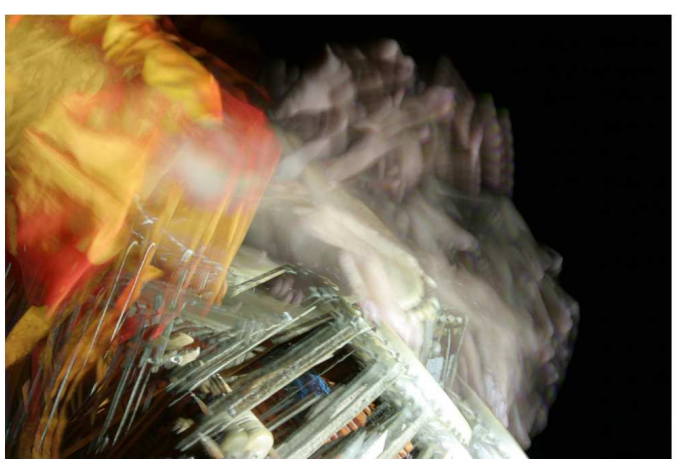

Image created by pixel maximum of all DLP photographs

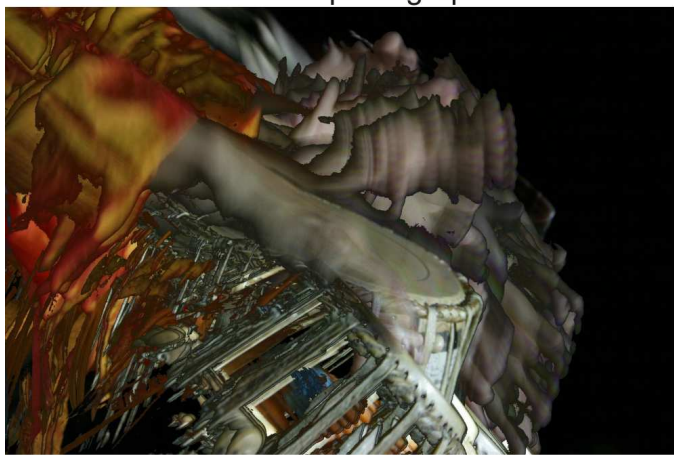

Image created by combining masked DLP photographs

(I) Tabla player summaries

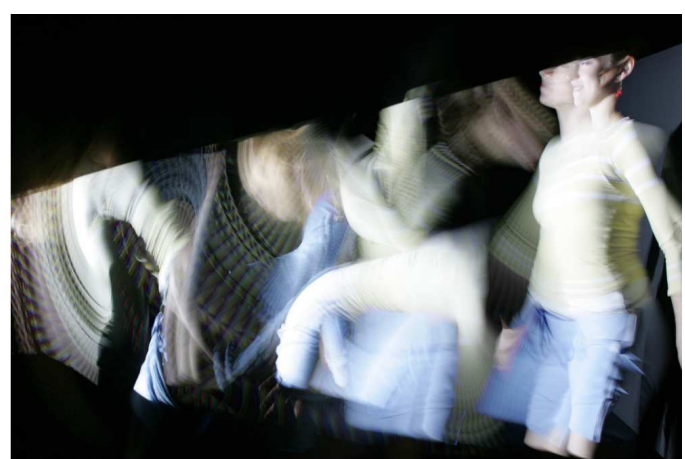

Image created by pixel maximum of all DLP photographs

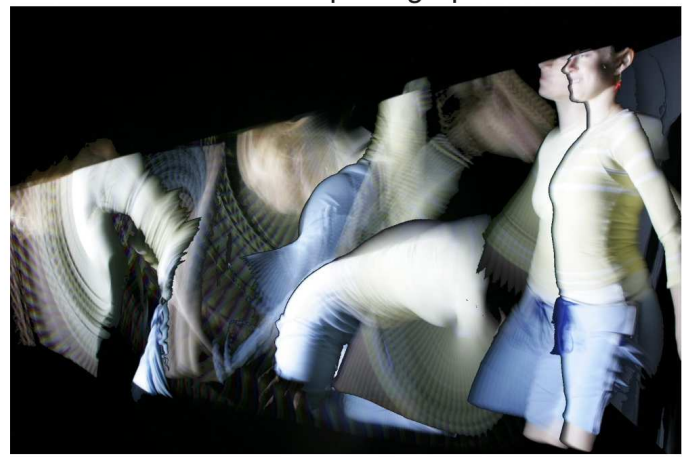

Image created by combining masked DLP photographs

(II) Ballet dancer summaries

Fig. 14 Colorwheel motion summaries: By combining DLP photographs in different ways we can summarize events. For both the tabla player, (a), and the ballet dancer, (b), we show summaries created by taking pixel maxima as well as by masking and superimposing the images. In the maxima case, no image ordering exists and all edges are blended. In the masking case, image order matters and edges exist between different stages of the action.

tween projectors of different manufacture. Still, we believe it would be useful to perform a broad empirical evaluation of a particular case, involving the InFocus IN38 DLP projector as observed by a Photron PCI1024 high-speed camera. In this section, we perform such experiments to investigate two important temporal dithering characteristics.

First, the dithering codes must be reliable and should not change arbitrarily. This would enable robust behavior for a vision system calibrated with a particular projector. Secondly, there must not be a large extent of overlap or redundancy in the dithering codes since this will constraint the possible set of illumination patterns. Finally, we will discuss a brute-force method to find incident illumination patterns from the measured profiles. We will begin here by describing experiments investigating the reliability and redundancy of temporal dithering codes:

Reliability: The temporal dithering is stable and repeatable but varies for each projector-camera system. Figure 16 investigates the consistency of the pattern for pixel intensity 250 at $10000 \mathrm{~Hz}$. We can see that the code varies periodically and repeats itself. This tempo- ral dithering is the code that the projector uses to represent the intensity 250 . We can also observe that the standard deviation of the repeated values is not high ( $\leq \frac{1}{5}$ of the intensity). This means that the temporal dither that we measure is reliable, in the sense that it preserves maxima and minima (peaks and troughs) from cycle to cycle. We have found that this holds for all the 255 pixel values for frame-rates of $10000 \mathrm{~Hz}, 3000 \mathrm{~Hz}$ and a $500 \mathrm{~Hz}$.

In addition to the repeatability of the dithering, we have also found dynamic range issues in this particular projector-camera pair. The image shown in Figure 1 is dark for the input brightness range of 0 to 90 . Despite the claim from manufacturers that the projector displays 8-bits of information, only about 160 patterns are usable for our experiments. To compensate for this, the projector performs spatial dithering in addition to temporal dithering in a few pixel blocks. This is an almost random effect that is not possible to reverse engineer without proprietary information from the manufacturers. We smooth out this effect by simply averaging over a small neighborhood for our processing. 


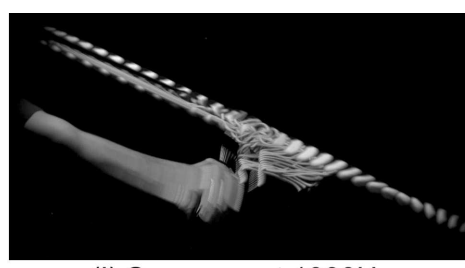

(I) Summary at $1000 \mathrm{~Hz}$ under fluorescent illumination

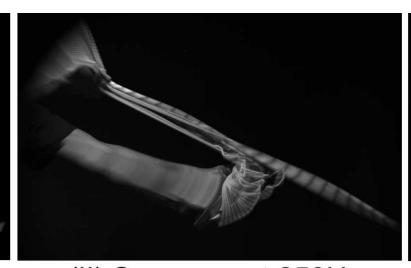

(II) Summary at $250 \mathrm{~Hz}$ under fluorescent illumination

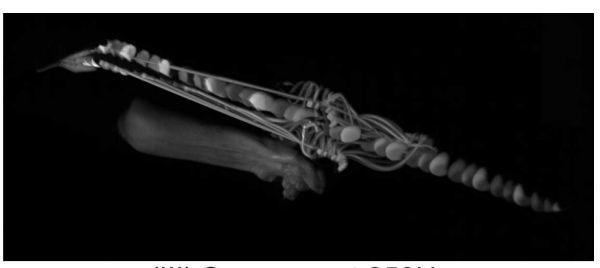

(III) Summary at $250 \mathrm{~Hz}$ under DLP illumination

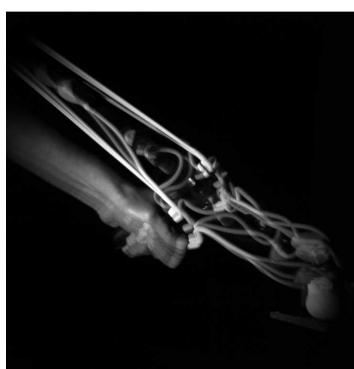

(a) Impact

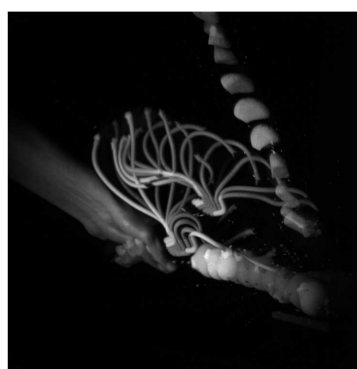

(b) Ricochet

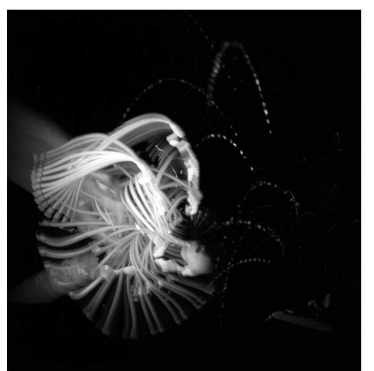

(c) Backlash

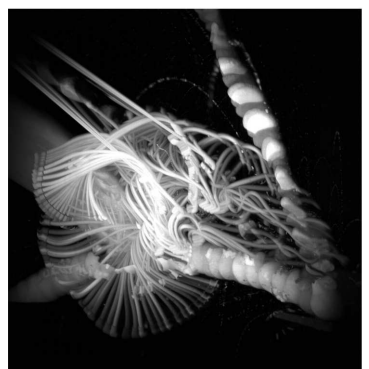

(d) Full Event

(IV) Summaries of different parts of a collision created with a sling-shot

Fig. 15 DMD motion summaries: Here we show experiments with a slingshot, which moves fast enough that the strobing effects are due to the DMD dithering and not the colorwheel. In the top row we demonstrate how DLP illumination allows the image capture of dynamic scenes with a low frame rate camera. In the bottom row we show how applying the method of [9], allows the summary of different aspects of a fast moving event, again with a low-frame rate camera.

Redundancy: In Figure 17, we show confusion matrices for all 256 patterns for three frame rates and for three metrics, euclidean, dot-product and KL-divergence. From the strong diagonal in these matrices, it is clear that each pixel has a unique encoding, and the profiles for three randomly selected intensities in the fifth column demonstrate this. The independence of these profiles is expected, since otherwise the projector would not be able to produce different emitted intensities. However, if we observe the fourth column in the figure, where one of the confusion matrices is thresholded, we observe that while most of the energy in the matrices is along the diagonal, there are many off diagonal elements, implying that clusters exist in the pattern. An intuitive explanation of this is that since the dithering code is integrated to provide the final pixel intensity, only a few mirror states need to be altered to move from one pixel intensity to one that is only slightly greater or lesser.

The reliability of the temporal codes opens up their use in active vision applications. However, the implications of their redundancy require careful selection of the codes and the illumination pattern that they imply. In this paper, we have empirically selected patterns, and we leave a more principled analysis for future work. However, we can suggest a simple brute-force approach for pattern design.

Designing an illumination pattern through brute-force search: Consider the toy problem in Fig 18 , where we wish to project a $2 \times 2$ alternating checker- board pattern onto the scene. Since the diagonal intensities of the checkerboard must be consistent, we must choose two values to make up the $2 \times 2$ checker board. At the right of the figure, we show the profiles for the intensities 200 and 230 at $10 \mathrm{kHz}$. In the 100 frame cycle, there are only two frames where the projected intensities at different enough to appear as a checkerboard pattern. If we pick these, we obtain the desired checkerboard pattern, but at a reduced $100 \mathrm{~Hz}$. The bottom row shows the projected intensities on a scene for these two frames, showing the checkerboard pattern.

Therefore to obtain a desired pattern, we must first, pick the static pattern to project (in the previous case it was alternating squares of value 200 and 230) and second, pick the frames to consider (such as frames 1 and 100 in the figure). Let the desired pattern be $A_{P Q R}$, which is essentially an $R$ length sequence of $P \times Q$ frames. In the previous example, this would be $A_{222}$ where the $2 \times 2$ pattern at $R=1$ is a checkerboard and the $2 \times 2$ pattern at $R=2$ is the inverse checkerboard. Now consider the dithering codes, as arranged in Figure 1(e). That image can be denoted as an array $T_{i j}$ where $i$ is the pattern index $(0-255)$ and $j$ is the frame number in the periodic cycle (for $10 \mathrm{kHz}$ in the figure, $j$ varies from 1 to 100). We wish to extract $A_{P Q R}$ from $T_{i j}$.

First we must compute the static pattern to project. Since there are $P \times Q$ slots, there are $255^{P Q}$ choices. If we can reduce these choices, by enforcing a spatial relationship between the patterns (such as alternate squares 


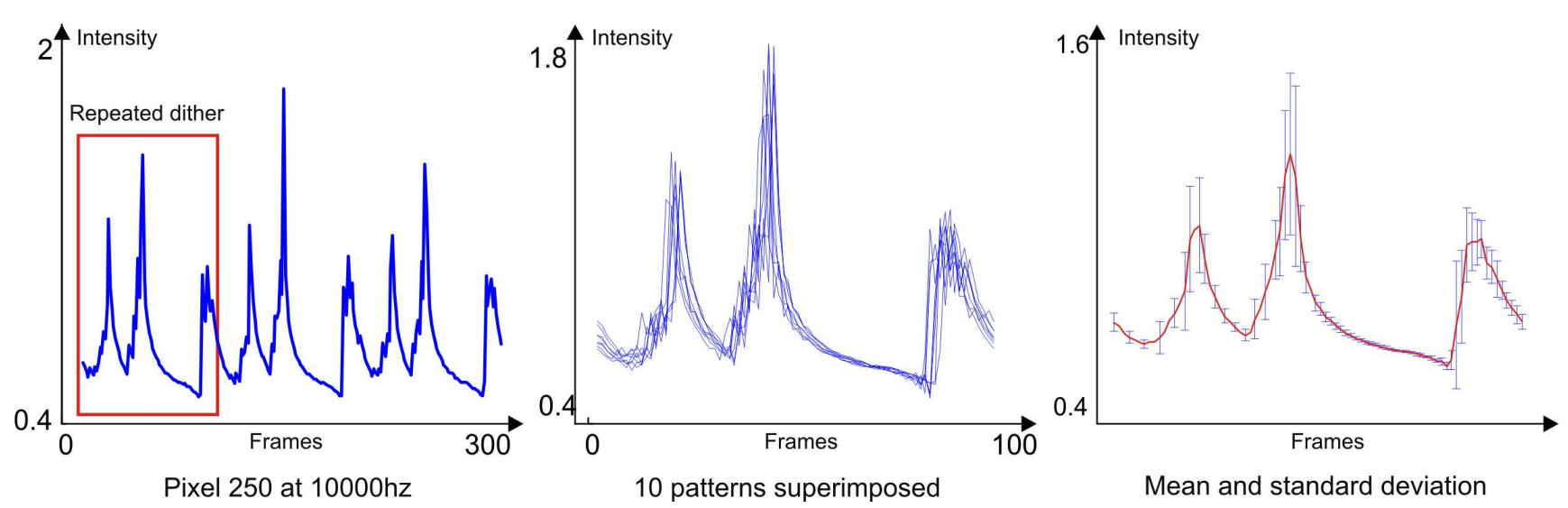

Fig. 16 How reliable is the dithering code?: In the first column we show the temporal dithering observed at a pixel of intensity 250 , captured at $10000 \mathrm{~Hz}$. Note that after a few frames the illumination code starts to repeat. We accumulated ten such patterns and superimposed them on each other, shown in the center column. These qualitatively show that the dithering code is consistent. A quantitative measure showing standard deviation is shown in the third column, and similar results hold for other pixel intensities at different frame-rates.

must be identical), we have a reduced space. For the checkerboard example, this is $255^{2}$. Every candidate in such a reduced exponential space creates a pattern of size $\operatorname{Px} Q \mathrm{x} N$, where $N$ is the periodicity, and for $10 \mathrm{kHz}$ is $N=100$.

From each candidate pattern of size $P \mathrm{x} Q \mathrm{x} N$, we must specify the $R \leq N$ frames from these such that the resulting, smaller sub-set best matches $A_{P Q R}$. Since no two frames can share the same $N$, this takes (using combination notation) ${ }_{n} C_{r}$ steps, if order in $A$ is unimportant. This scenario is true for many applications such as photometric stereo or illumination multiplexing. If the order in $A$ is important, then each selection of $r$ reduces the space of possible further frames. This can be denoted by a summation of permutations given by $\Sigma_{i n_{i}} C_{r}$ starting with $n_{1}=n$. A brute force method would be to calculate the SSD distance between $A_{P Q R}$ and these candidates, and select the candidate with the smallest error.

Detecting the illumination pattern: Consider an active vision system for a dynamic scene where the fastest object in the scene moves with an image-plane speed $v$, the frame rate of the camera is $f$ and $n$ is the minimum number of images required to extract some scene property. For example in classical lambertian photometric stereo, we require three images under non-collinear distant lighting and $n=3$. One way to extend such techniques to dynamic scenes is to rapidly vary the lighting such that the apparent object motion in the time interval $\frac{n}{f}$ is zero. Since we would like the object to appear to be static, we wish to minimize the error $e$ given by the object motion in the image plane, $e=v \cdot\left(\frac{n}{f}\right)$. To get effective performance we would ideally like $e$ to be zero or at least sub-pixel. Since we can- not control the object motion $v$ and, usually, the frame rate $f$ of the camera is fixed, we must vary $n$. However, $n$ depends on the requirement of the algorithm.

For example, in reconstruction algorithms, $n$ must be large enough to enable correspondence matching. There exists a trade-off between the number of frames needed for robust matching (larger $n$ ) and the ability to reconstruct fast objects ( $\operatorname{smaller} n$ ). A simple simulation using the codes taken at $10000 \mathrm{~Hz}$ is shown in Figure 19, where the confusion matrices become less diagonal as the number of frames becomes low. One final issue is the ${ }_{n} C_{r}$ selection step in the pattern design. As shown in Figure 18, a $10000 \mathrm{~Hz}$ camera reduces to a $100 \mathrm{~Hz}$ system, since a checkered pattern was required. Therefore the choice of pattern can render most of the intensities in the dithering codes as 'useless' and reduces the effective frame rate of the system by the number of correct matches found. Such wasted ditherings are shown by the gaps between the yellow vertical lines in the graph in Figure 18.

\section{Discussion}

We have demonstrated that illumination from DLP projectors shows flickering or dithering due to the effect of the DMD chip and shown how to exploit this effect to extend active vision techniques to dynamic scenes. We conclude here by providing a brief discussion of some trade-offs and limitations of our technique:

Speed vs. accuracy trade-off: One limitation of our approach is the requirement of a high speed camera. The acquisition speed of the camera and the effective speed of performance achieved depend on the task at hand and the signal-to-noise ratio of the captured im- 


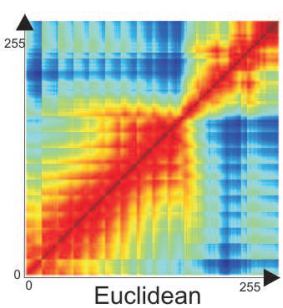

Euclidean

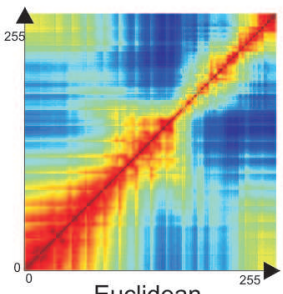

Euclidean

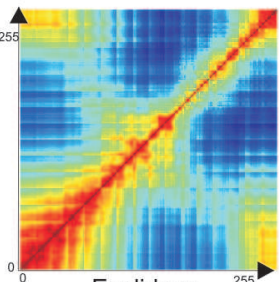

Euclidean

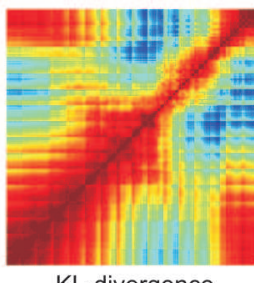

KL-divergence

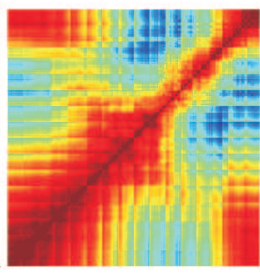

KL-divergence

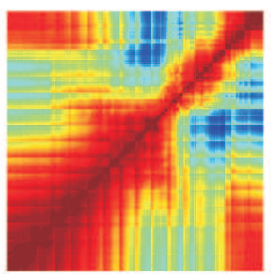

KL-divergence

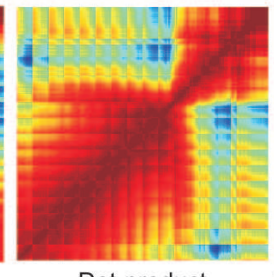

Dot-product

(a) $500 \mathrm{hz}$

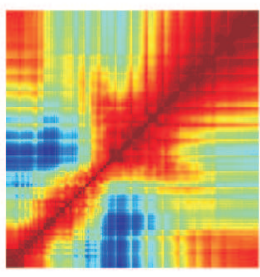

Dot-product

(b) $3000 \mathrm{hz}$

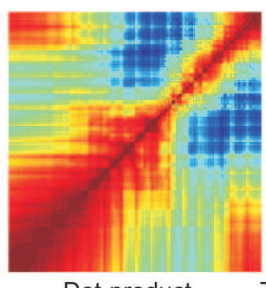

Dot-product

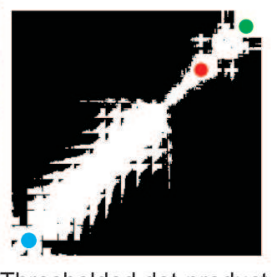

Thresholded dot-product

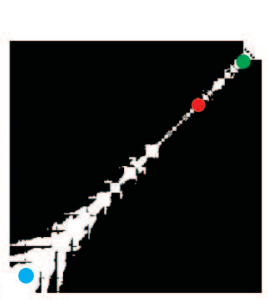

Thresholded dot-product

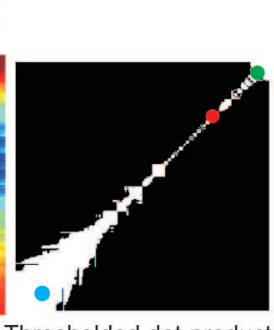

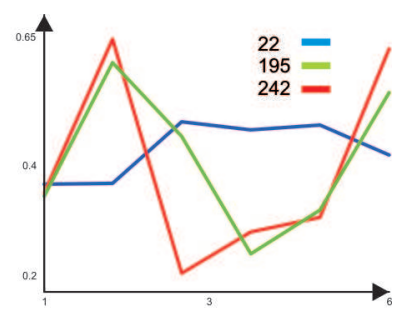
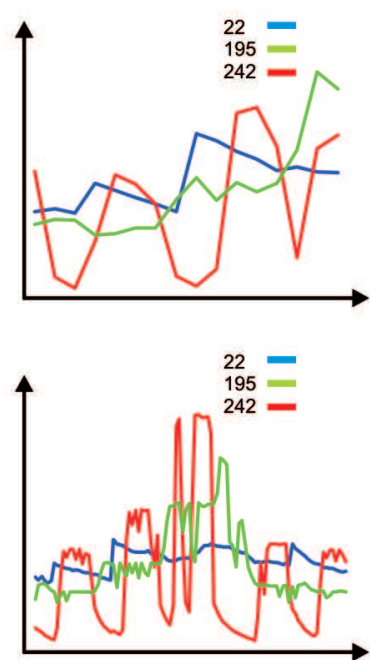

(c) $10000 \mathrm{hz}$

Fig. 17 Confusion matrices for temporal dithering patterns: Here we show the confusion matrices for 255 emitted intensities, for three distance metrics. Reddish colors imply a high value while blueish colors denote a lower value. Note that while the matrices are mostly diagonal, there exist large off-diagonal patches of high correlation. This is very evident from the thresholded matrices, which show strong off diagonal components. The smoothing effect of camera integration can be seen with the trend from top to bottom, where low camera frame-rate smooths out differences creating stronger clustering, whereas high-frame rate creates stronger diagonal responses. Overall, these matrices show that the dithering codes are independent, which is expected since these denote different projected pixels. The profiles of three randomly selected pixel intensities are plotted, showing their differences.

ages. For instance, the decision to use 10 frames for demultiplexing illumination or photometric stereo, or to use 20 frames for structured light, was mainly influenced by the noise characteristics of the camera. A more scientific exploration of this trade-off is required to better understand the benefits of our approach to each technique. A future avenue of research is to design 2D spatial intensity patterns that create temporal dithering codes that are optimal for the task at hand.

Extensions to flutter-flash and other illumination modulations: We believe that the temporal illumination dithering can be applied to a broader range of methods including pixel-wise optical flow estimation and tracking, projector defocus compensation and depth from defocus [50] and spectral de-multiplexing. While we exploit the temporal dithering already built-in to the projector, we do not have a way of controlling it explicitly. Better control is obtained by using a more expensive and special high speed MULE projector [20], per- haps for applications where the strobing varies across time, such as in Figure 20. Strobe lighting, fast LED [27] and flash modulation are also effective in temporally varying (not dithering) the illumination.

Implementation Issues: The selection of the pattern decides the frame-rate at which reconstruction can occur. This can easily be done using the calibration method of Figure 1. However, non-DMD factors such as projector heat, time-cycle, color-wheel start position etc seem to severely effect the calibration. Therefore, we suggest that the pattern be selected and used during or just previous to the experiment. Since we are dealing with active vision techniques, ambient light may cause issues with low dynamic range cameras. All our experiments were performed in the dark, but DLP projectors are bright enough to be detected in a variety of settings, such as underground, underwater and outdoor scenes at night. The most powerful DLP projectors for cinema can project over several meters, and therefore our techniques have application for a variety of long-range 


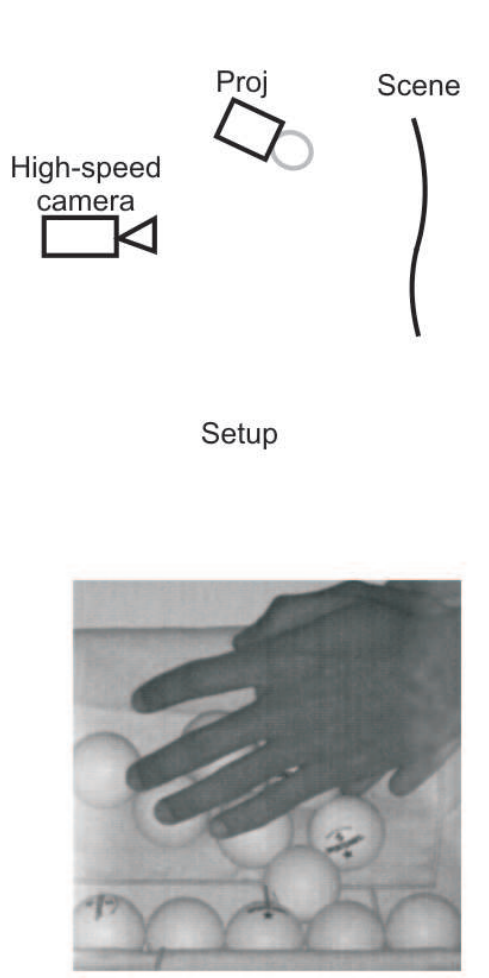

Scene

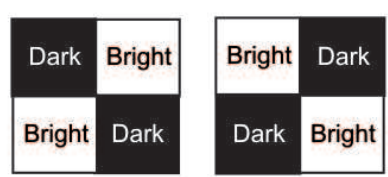

Desired Pattern

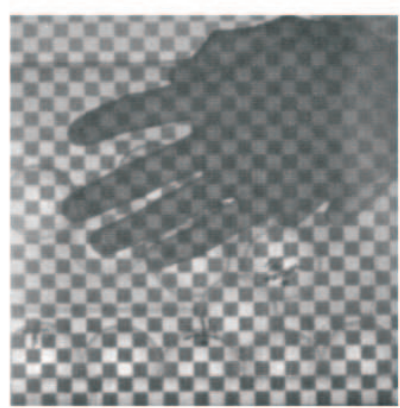

Frame 1

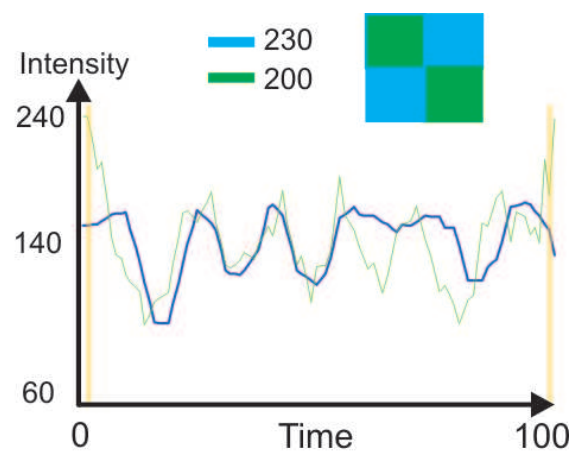

Profiles with desired frames (yellow)

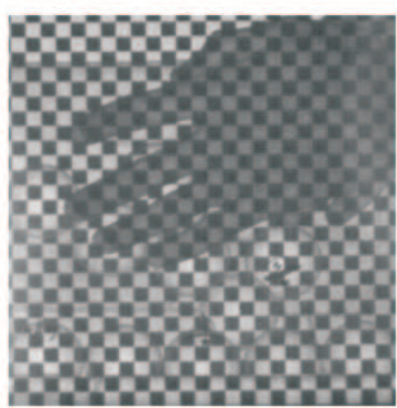

Frame 100

Example scene illuminated by a $200 / 230$ checkerboard pattern

Fig. 18 Patterns selected by brute-force for temporal dithering at 10000Hz: If we desire a particular ordered pattern as shown in the top row, where a single projector sends out a checkerboard pattern, we need to find two intensities to fit the pattern. The profiles of the two selected intensities are shown, and they occur in the required configuration only twice, allowing scenes that move only at $100 \mathrm{~Hz}$. The bottom row shows two frames of the pattern, projected onto a scene.

tasks. However, for applications requiring even longer ranges, passive techniques should be used instead.

In summary, in this work, we have discussed how to calibrate the dithering patterns from an off-the-shelf DLP projector using a high-speed camera. We have noticed that dithering is only observable with cameras of frame-rates of $250 \mathrm{~Hz}$ and higher, which are getting more reasonably priced (currently around $\$ 1000$ ). The dithering codes have been shown to be both reliable, but contain a redundancy that limits the types of patterns that are possible. We demonstrated a brute-force search method to design the slide that must be sent to the projector, and discussed the implications for the speed of the scene that can be handled by the system. We have also shown applications of temporal dithering on well-known active vision methods, such as structured light reconstruction, illumination demultiplexing, photometric stereo and high-frequency strobe photography.

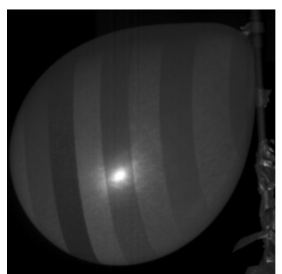

Fully illuminated

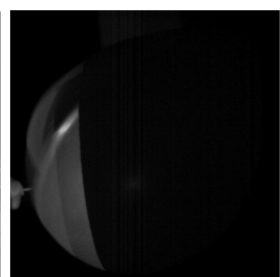

Start of balloon burst Striped pattern at $10000 \mathrm{~Hz}$

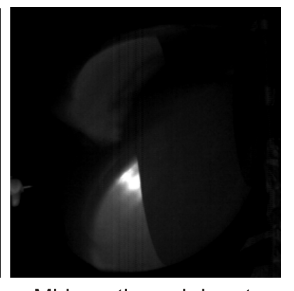

Midway through burst
Fig. 20 DLP illumination as a programmable aperture: On the left we show a balloon illuminated by a striped pattern from a DLP projector. Each stripe dithers at a different rate. We show two instances just after the balloon is burst, showing the edge of the contracting balloon in two different positions. If the pattern was uniformly set to either the first or second stripe value, one of these events would have been missed.

\section{Acknowledgements}

This research was supported in parts by ONR grants N00014-08-1-0330 and DURIP N00014-06-1-0762, and NSF CAREER award IIS-0643628. The authors thank the anonymous reviewers for their useful comments. 


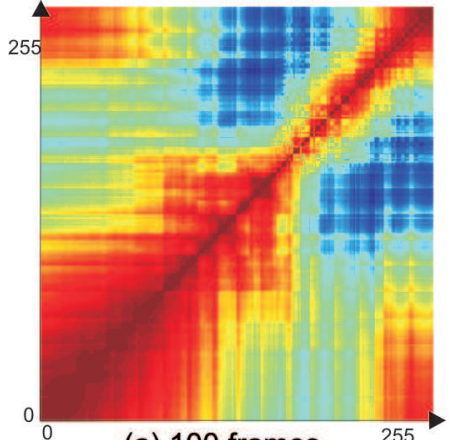

(a) 100 frames

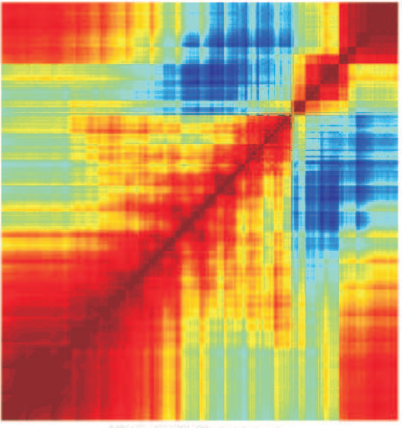

(b) 50 frames

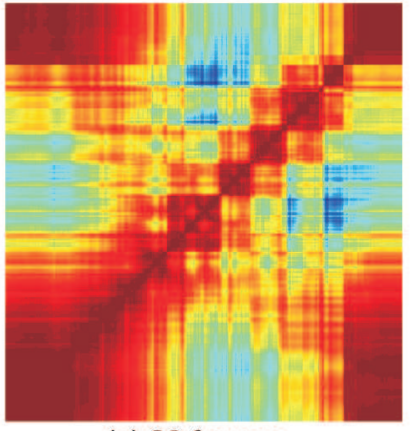

(c) 20 frames

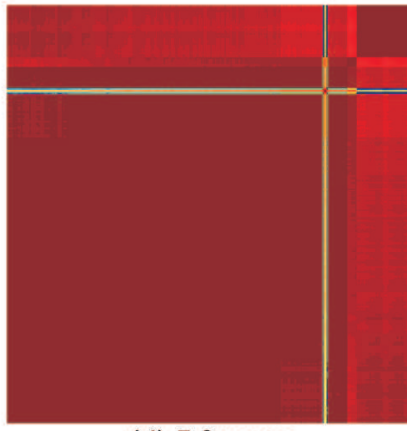

(d) 5 frames

Confusion matrices at $10000 \mathrm{hz}$

Fig. 19 Diagonality of confusion matrices as a function of frame-rate: We simulate reduced frame-rate by first sampling the dithering code at $10000 \mathrm{~Hz}$ and then dropping every 50th, 20th and 5th frame. Note that this excludes the smoothing effect of camera integration, which further reduces the diagonality of the matrices. The figure shows that the fastest scene on which this code is detectable is at $\frac{10000}{20}=500 \mathrm{~Hz}$.

\section{References}

1. Paul J Besl. Active, optical range imaging sensors. Machine Vision and Applications, 1(2):127-152, 1988.

2. James R. Bitner, Gideon Ehrlich, and Edward M. Reingold. Efficient generation of the binary reflected gray code and its applications. Communications of the ACM, 19(9):517 - 521, 1976.

3. K. L. Boyer and A. C. Kak. Color-encoded structured light for rapid active ranging. IEEE Transactions on Pattern Analysis and Machine Intelligence, 9(1):14-28, 1987.

4. Q. Chen and T. Wada. A light modulation/demodulation method for real-time $3 \mathrm{~d}$ imaging. $3 \mathrm{D}$ digital imaging and modeling, 2005.

5. D. Cotting, M. Naef, M. Gross, and H. Fuchs. Embedding imperceptible patterns into projected images for simultaneous acquisition and display. In ISMAR, 2004.

6. B. Curless and M. Levoy. Better optical triangulation through spacetime analysis. ICCV, 1995.

7. J. Davis, D. Nehab, R. Ramamoothi, and S. Rusinkiewicz. Spacetime stereo : A unifying framework for depth from triangulation. IEEE CVPR, 2003.

8. D. Dudley, W. Duncan, and J. Slaughter. Emerging digital micromirror device (dmd) applications. Proc. of SPIE, 4985, 2003.

9. W. Freeman and H. Zhang. Shape-time photography. CVPR, 2003.

10. Olaf Hall-Holt and Szymon Rusinkiewicz. Stripe boundary codes for real-time structured-light range scanning of moving objects. In Proc. the Eighth International Conference on Computer Vision, volume 2, pages 359-366, 2001.

11. A. Hertzmann and S. M. Seitz. Shape and materials by example: A photometric stereo approach. IEEE CVPR, 2003.

12. Eli Horn and Nahum Kiryati. Toward optimal structured light patterns. Image and Vision Computing, 17:87-97, 1999.

13. T. Jarvenpaa. Measuring color breakup of stationary images in field-sequential-color displays. Journal of the Society for Information Display, 2005.

14. A. Jones, I. McDowall, H. Yamada, M. Bolas, and P. Debevec. Rendering for an interactive 360 degree light field display. In ACM SIGGRAPH, 2007.

15. S. Kima, T. Shibataa, T. Kawaia, and K. Ukaib. Ergonomic evaluation of a field-sequential colour projection system. Displays, 2007.
16. S. J. Koppal and S. G. Narasimhan. Illustrating motion through dlp photography. PROCAMS, 2009.

17. S.J. Koppal, S. Yamazaki, and S. Narasimhan. Dlp photograph website. http://www.cs.cmu.edu/ ILIM/projects/IM/procams webpage/motion_illustrations.html, 2010.

18. S.J. Koppal, S. Yamazaki, and S. Narasimhan. Temporal dithering website. http://www.cs.cmu.edu/ ILIM/projects/IL/dlp-dithering/, 2010.

19. J. Lee, S. Hudson, J. Summet, and P. Dietz. Moveable interactive projected displays using projector based tracking. Symposium on User Interface Software and Technology, 2005.

20. I. McDowall and M. Bolas. Fast light for display, sensing and control applications. In IEEE VR Workshop on Emerging Display Technologies, 2005.

21. T. Miyasaka, K. Kuroda, M. Hirose, and K. Araki. High speed 3-d measurement system using incoherent light source for human performance analysis. In Proc. the 19th Congress of The International Society for Photogrammetry and Remote Sensing, pages 65-69, 2000.

22. M. Mori, T. Hatada, K. Ishikawa, T. Saishoji, O. Wada, J. Nakamura, and N. Terashima. Mechanism of color breakup in field-sequencial-color projectors. Journal of the Society for Information Display, 1999.

23. Hiroyoshi Morita, Kazuyasu Yajima, and Shojiro Sakata. Reconstruction of surfaces of 3 -d objects by m-array pattern projection method. In Proc. International Conference on Computer Vision, pages 468-473, 1988.

24. Srinivasa G Narasimhan, Sanjeev Jagannatha Koppal, and Shuntaro Yamazaki. Temporal dithering of illumination for fast active vision. In Proc. European Conference on Computer Vision, October 2008.

25. S. K. Nayar, V. Branzoi, and T. Boult. Programmable imaging using a digital micromirror array. IEEE CVPR, 2004.

26. S. K. Nayar, G. Krishnan, M. D. Grossberg, and R. Raskar. Fast separation of direct and global components of a scene using high frequency illumination. ACM SIGGRAPH, 2006.

27. H. Nii, M. Sugimoto, and M. Inami. Smart light-ultra high speed projector for spatial multiplexing optical transmission. In IEEE PROCAMS, 2005.

28. K. Ishikawa O. Wada, J. Nakamura and T. Hatada. Analysis of color breakup in field-sequential color projection system for large area displays. Display Workshops, 1999. 
29. M. Ogata, K. Ukai, and T. Kawai. Visual fatigue in congenital nystagmus caused by viewing images of color sequential projectors. Display Technology, 2005.

30. O. Packer, L. Diller, J. Verweij, B. Lee, J. Pokorny, D. Williams, D. Dacey, and D. H. Brainard. Characterization and use of a digital light projector for vision research. Vision Research, 2001.

31. J.L. Posdamer and M.D. Altschuler. Surface measurement by space-encoded projected beam system. Comput. Vision Graphics Image Processing, 18(1), January 1982.

32. R. Raskar, A. Agrawal, and J. Tumblin. Coded exposure photography: Motion deblurring using fluttered shutter. $A C M$ SIGGRAPH, 2006.

33. R. Raskar, G. Welch, M. Cutts, A. Lake, L. Stesin, and H. Fuchs. The office of the future : A unified approach to image-based modeling and spatially immersive displays. In ACM SIGGRAPH, 1998.

34. Ramesh Raskar and Paul Beardsley. A self-correcting projector. In Proc. Computer Vision and Pattern Recognition, pages 504-508, 2001.

35. Joaquim Salvi, Jordi Pages, and Joan Batlle. Pattern codification strategies in structured light systems. Pattern Recognition, 37(4):827-849, 2004.

36. Kosuke Sato and Seiji Inokuchi. Range-imaging system utilizing nematic liquid crystal mask. In Proc. International Conference on Computer Vision, pages 657-661, 1987.

37. D. Scharstein and R. Szeliski. High-accuracy stereo depth maps using structured light. In $C V P R, 2003$.

38. Y. Y. Schechner, S. K. Nayar, and P. N. Belhumeur. A theory of multiplexed illumination. ICCV, 2003.

39. P. Sen, B. Chen, G. Garg, S. R. Marschner, M. Horowitz, M. Levoy, and H. P. A. Lensch. Dual photography. ACM SIGGRAPH, 2005.

40. D. Takhar, J. Laska, M. Wakin, M. Duarte, D. Baron, S. Sarvotham, K. Kelly, and R. Baraniuk. A new compressive imaging camera architecture using optical-domain compression. In Computational Imaging IV at SPIE Electronic Imaging, 2006.

41. E. Umezawa, T. Shibata, T. Kawai, and K. Ukai. Ergonomic evaluation of the projector using color-sequential display system. 45th Annual Congress of the Japan Ergonomics Assoc., 2004.

42. Thibaut Weise, Bastian Leibe, and Luc Van Gool. Fast 3d scanning with automatic motion compensation. In Proc. Computer Vision and Pattern Recognition, pages 1-8, June 2007.

43. A. Wenger, A. Gardner, C. Tchou, J. Unger, T. Hawkins, and P. Debevec. Performance relighting and reflectance transformation with time-multiplexed illumination. ACM SIGGRAPH, 2005.

44. P. M. Will and K. S. Pennington. Grid coding: A preprocessing technique for robot and machine vision. AI, 2, 1971.

45. R.J. Woodham. Photometric method for determining surface orientation from multiple images. OptEng, 19(1), 1980.

46. Y. You and M. Kaveh. A regularization approach to joint blur identification and image restoration. Transactions on image processing, 1996.

47. M. Young, E. Beeson, J. Davis, S. Rusinkiewicz, and R. Ramamoorthi. Viewpoint-coded structured light. IEEE CVPR, 2007.

48. L. Zhang, B. Curless, and S. M. Seitz. Rapid shape acquisition using color structured light and multi-pass dynamic programming. 3DPVT, 2002.

49. L. Zhang, B. Curless, and S. M. Seitz. Spacetime stereo: Shape recovery for dynamic scenes. IEEE CVPR, 2003.

50. L. Zhang and S. K. Nayar. Projection defocus analysis for scene capture and image display. ACM SIGGRAPH, 2006.
51. Zhengyou Zhang. A flexible new technique for camera calibration. IEEE Transactions on Pattern Analysis and Machine Intelligence, 22(11):1330-1334, 2000.

52. T. Zickler, P. Belhumeur, and D. J. Kriegman. Helmholtz stereopsis: Exploiting reciprocity for surface reconstruction. ECCV, 2002. 